\title{
Protective Effects of
}

Dexmedetomidine on the Vascular Endothelial Barrier Function by Inhibiting Mitochondrial Fission via ER/Mitochondria Contact

\section{OPEN ACCESS}

Edited by:

Fabio DiLisa,

University of Padua, Italy

Reviewed by:

Baolin Liu,

China Pharmaceutical University,

China

Li Guobing,

Army Medical University, China

*Correspondence:

Liangming Liu

liangmingliu@yahoo.com

Yi Hu

Huyi921@sina.com

Tao $\mathrm{Li}$

Lt200132@163.com

${ }^{\dagger}$ These authors have contributed equally to this work

Specialty section:

This article was submitted to

Mitochondrial Research,

a section of the journal

Frontiers in Cell and Developmental

Biology

Received: 01 December 2020

Accepted: 23 February 2021

Published: 11 March 2021

Citation:

She H, Zhu Y, Deng H, Kuang L,

Fang $H$, Zhang $Z$, Duan $C$, Ye J,

Zhang J, Liu L, Hu Y and Li T (2021)

Protective Effects

of Dexmedetomidine on the Vascular

Endothelial Barrier Function by

Inhibiting Mitochondrial Fission via

ER/Mitochondria Contact.

Front. Cell Dev. Biol. 9:636327.

doi: 10.3389/fcell.2021.636327

\author{
Han She ${ }^{1}$, Yu Zhu' ${ }^{2}$, Haoyue Deng ${ }^{2}$, Lei Kuang' ${ }^{2}$, He Fang ${ }^{1}$, Zisen Zhang ${ }^{2}$, \\ Chenyang Duan ${ }^{2}$, Jiaqing $\mathrm{Ye}^{1}$, Jie Zhang ${ }^{2}$, Liangming $\mathrm{Liu}^{2 * t}$, Yi Hu ${ }^{1 *}$ and Tao $\mathrm{Li}^{2 * \dagger}$ \\ 1 Department of Anesthesiology, Daping Hospital, Army Medical University, Chongqing, China, ${ }^{2}$ State Key Laboratory \\ of Trauma, Burns and Combined Injury, Second Department of Research Institute of Surgery, Daping Hospital, Army Medical \\ University, Chongqing, China
}

The damage of vascular endothelial barrier function induced by sepsis is critical in causing multiple organ dysfunctions. Previous studies showed that dexmedetomidine (Dex) played a vital role in protecting organ functions. However, whether Dex participates in protecting vascular leakage of sepsis and the associated underlying mechanism remains unknown yet. We used cecal ligation and puncture induced septic rats and lipopolysaccharide stimulated vascular endothelial cells (VECs) to establish models in vivo and in vitro, then the protective effects of Dex on the vascular endothelial barrier function of sepsis were observed, meanwhile, related mechanisms on regulating mitochondrial fission were further studied. The results showed that Dex could significantly reduce the permeability of pulmonary veins and mesenteric vessels, increase the expression of intercellular junction proteins, enhance the transendothelial electrical resistance and decrease the transmittance of VECs, accordingly protected organ functions and prolonged survival time in septic rats. Besides, the mitochondria of VECs were excessive division after sepsis, while Dex could significantly inhibit the mitochondrial fission and protect mitochondrial function by restoring mitochondrial morphology of VECs. Furthermore, the results showed that ER-MITO contact sites of VECs were notably increased after sepsis. Nevertheless, Dex reduced ER-MITO contact sites by regulating the polymerization of actin via $\alpha_{2}$ receptors. The results also found that Dex could induce the phosphorylation of the dynamin-related protein 1 through down-regulating extracellular signal-regulated kinase1/2, thus playing a role in the regulation of mitochondrial division. In conclusion, Dex has a protective effect on the vascular endothelial barrier function of septic rats. The mechanism is mainly related to the regulation of Drp1 phosphorylation of VECs, inhibition of mitochondrial division by ER-MITO contacts, and protection of mitochondrial function.

Keywords: sepsis, Drp1, ER-MITO contact, dexmedetomidine, vascular endothelial barrier function 


\section{INTRODUCTION}

Sepsis is a life-threatening organ dysfunction caused by a dysregulated host response to infection (Jawad et al., 2012; Peake et al., 2014). The damage of vascular endothelial barrier function is a critical pathophysiology process during the development of sepsis (Koh et al., 2010), which will lead to multiple organ dysfunction syndrome (MODS) with high mortality. The vascular endothelium is an important organ of the body, which constitutes the fundamental barrier between blood and tissues (Page and Liles, 2013; Zheng et al., 2020). However, there is still lack of targeted prevention and treatment of vascular leakage after sepsis. Therefore, it is of great clinical significance to look for effective measures to improve sepsis-induced vascular endothelial barrier dysfunction.

Dexmedetomidine (Dex) is a highly selective $\alpha_{2}$-adrenoceptor agonist with sedative, analgesic, and anxiolytic effects (Nelson et al., 2003). Compared with midazolam or propofol, Dex can simulate "natural sleep" without distinct respiratory depression and is suitable for critically ill patients. Previous studies showed that Dex had a crucial protective effect on the damage of many organs, such as lungs and intestines, by inhibiting inflammation and regulating oxidative stress (Sha et al., 2019). However, whether Dex could protect the vascular endothelial barrier function in sepsis and how it works remains obscure.

Dysfunction of mitochondria is a major factor contributing to organ failure, and the dysfunction degree of mitochondria is directly related to the outcome of patients (Brooks et al., 2009; Liu et al., 2012; Roy et al., 2019). Recent studies found that Dex could protect organ function by recovering mitochondrial function. For example, Dex could protect against cerebral ischemia-reperfusion injury by activating mitochondrial ATP potassium channels (Yuan et al., 2017). Additionally, basic research demonstrated that the dynamic balance of mitochondria, including mitochondrial fission and fusion, ensured mitochondrial function maintenance under physiological conditions. While pathological stimulation could induce overactive fission of mitochondria (Hall et al., 2013), which seriously impairs mitochondrial function, resulting in decreased ATP production, cellular calcium disorder, and MPTP opening. However, it is still unknown whether Dex can protect the vascular endothelial barrier function of septic rats by regulating mitochondrial fission.

Herein, we used the cecal ligation and puncture (CLP) induced septic rats and lipopolysaccharide (LPS) stimulated vascular endothelial cells (VECs) to establish models in vivo and in vitro, and explored the protective effect of Dex on the vascular endothelial barrier function of sepsis and the underlying mechanism associated with mitochondrial fission.

\section{MATERIALS AND METHODS}

\section{Ethical Approval of the Study Protocol}

All procedures were performed under the guidelines for the Care and Use of Laboratory Animals published by the US National Institutes of Health and were approved by the Laboratory
Animal Welfare and Ethics Committee of the Army Medical University (No. DHEC-2012-069). Sprague-Dawley (SD) rats were purchased from the Animal Center of the Research Institute of Surgery.

\section{Reagents}

Dexmedetomidine was purchased from Hengrui (Jiangsu, China). Albumin-fluorescein isothiocyanate conjugate (FITCBSA), Evans Blue, and Lipopolysaccharide (LPS) were purchased from Sigma (St. Louis, MO, United States). Antibodies for Drp1, ZO-1, VE-cadherin, Occludin, $\beta$-actin, ANT, and ROS Detection Kit were purchased from Abcam (Cambridge, MA, United States). Antibodies for phospho-Drp1 (Ser616 and Ser637), ERK1/2, phospho-ERK1/2 were purchased from Cell Signaling Technology (Danvers, MA, United States). MitoTracker and ER-Tracker were purchased from Thermo Fisher Scientific (Waltham, MA, United States). Mitochondria Isolation Kit was purchased from Invent Biotechnologies, Inc. (Beijing, CHINA). Atipamezole was purchased from MedChemExpress (Monmouth, NJ, United States). All other chemicals were purchased from Sigma unless specifically mentioned otherwise.

\section{Animals Preparation and Sepsis Model}

Adult male and female Sprague-Dawley (SD) rats (200-220 g) were anesthetized with sodium pentobarbital $(45 \mathrm{mg} / \mathrm{kg}$ intraperitoneal). CLP induced the sepsis model of rats with aseptic methods as described previously (Zhu et al., 2016). Briefly, alaparotomy was performed, and then the cecum was exposed and ligated. The hole was punctured $0.7 \mathrm{~cm}$ from the distal end with a triangular needle (the needle was approximately 1.5 $\mathrm{mm}$ indiameter). Feces were allowed to flow into the abdominal cavity. After closure of the abdomen, the rats were returned to the cages and allowed food and water ad libitum.

\section{Cell Preparation}

Vascular endothelial cells were obtained from pulmonary veins of SD rats, as described previously (Zhao et al., 2020). Rats were anesthetized and sterilized with iodine, and then rats received thoracotomy. The pulmonary veins were separated from hilus pulmonis after the heart was cut off. After washed with sterile PBS for 3 times, the veins were sheared to pieces, and attached to the bottom of the culture flask with $5 \mathrm{~mL}$ ECM (Scicell, America; $5 \%$ fetal bovine serum) medium. 3 days later, the pieces were removed from the flask, and the cells crawling on at the bottom of the culture flask were VECs, and the 3-5 passage of VECs were used in the following study.

\section{FITC-BSA Leakage of Mesenteric Microvessels}

Rats were anesthetized, and the ileocecal portion of the mesentery was exposed and placed in a transparent stage. The mesentery was moisturized with $37^{\circ} \mathrm{C}$ saline throughout the whole procedure to keep it warm and moist. Then rats were injected with FITCBSA $(9 \mathrm{mg} / \mathrm{kg})$ intravenously. 6 min after basal observation, fluorescence intensity of FITC-BSA in mesenteric microvessels 
was measured at $0,1,3$, and $6 \mathrm{~min}$ by inverted intravital microscopy (HAMAMATSU, Japan).

\section{Measurement of Pulmonary Vascular Permeability in Rats With FITC-BSA/Evans Blue}

Rats were anesthetized, and FITC-BSA $(9 \mathrm{mg} / \mathrm{kg}$ ) or Evans blue $(60 \mathrm{mg} / \mathrm{kg}$ ) was injected through the jugular vein. The abdomen was opened along with the linea alba abdominis after $1 \mathrm{~h}$ of FITC-BSA (or $30 \mathrm{~min}$ after Evans blue) administration. The abdominal aorta was cut, and the phosphate buffer solution (PBS) was perfused through the jugular vein. The left lung's upper lobe was dried and weighed, and PBS was added and homogenized in an ice-bath. The homogenate was transferred to a centrifuge $\left(8,000 \mathrm{~g}, 4^{\circ} \mathrm{C}, 10 \mathrm{~min}\right)$, and the supernatant was then centrifuged again $\left(16,000 \mathrm{~g}, 4^{\circ} \mathrm{C}, 10 \mathrm{~min}\right)$. The optical density (OD) of the supernatant was determined with a spectrophotometer [excitation wave length: $562 \mathrm{~nm}$ (Evans blue)]. Besides, the protein concentration of the supernatant was detected with the BCA protein assay kit (Thermo Fisher Scientific). Finally, the OD value ratio to the protein concentration was considered the pulmonary vascular permeability. Right lung tissue was embedded in Optimal Cutting Temperature compound, and frozen sections (10-20 mm thickness) were generated. The infiltration of FITC-BSA in the lung was observed by a laser confocal microscope (Leica SP5, Germany). The mean optical density was considered to reflect the infiltration of FITC-BSA in the lung. In the Evans blue group, the lung tissue was photographed with a camera (Pentax K7) before homogenization.

\section{Transendothelial Electrical Resistance(TER) and FITC-BSA Leakage of VECs}

VECs were seeded on six-well, $3 \mu \mathrm{m}$ cell culture inserts (BD Biosciences, Franklin Lakes, NJ, United States), and TER of VECs was assessed by Voltohmmetre (World Precision Inc., America) every $30 \mathrm{~min}$. The TER value measured in the noncell chamber was regarded as blank control. The resistivity of VECs $=$ (actual TER-blank control)/actual TER. FITC-BSA leakage of VECs was measured after TER analysis, FITC-BSA $(10 \mu \mathrm{g} / \mathrm{mL})$ was added into upper inserts of the transwell, and $200 \mu \mathrm{L}$ of the medium of the lower chamber at $10,20,30,40,50$, and $60 \mathrm{~min}$ was collected for the measurement of fluorescence intensity. An equal volume of culture medium was added into the lower chamber after medium each collection. FITC-BSA leakage $(\%)=(\mathrm{A} 10+\mathrm{A} 20+\mathrm{A} 30+\mathrm{A} 40+\mathrm{A} 50+\mathrm{A} 60) /$ total fluorescence intensity, and Ax represented the fluorescence intensity at $\mathrm{x}$ min.

\section{Transmission Electronic Microscopy Observation}

Fresh pulmonary veins were quickly fixed with arsenate buffer containing $2.5 \%$ glutaraldehyde $\left(\mathrm{pH}=7.4,4^{\circ} \mathrm{C}\right)$ for $24 \mathrm{~h}$. After three $5 \mathrm{~min}$-wash with $0.13 \mathrm{M} \mathrm{PBS}$, the veins were postfixed in $1 \% \mathrm{OsO}_{4}$ for $2 \mathrm{~h}$ at room temperature and then dehydrated in a graded ethanol series $(65,70,75,80$, and $95 \%$ for 10 min each)
(Duan et al., 2020). After that, the veins were incubated with tertbutoxide for $10 \mathrm{~min}$ and then dried with $\mathrm{CO}_{2}$, stained with uranyl acetate, coated with gold $(\mathrm{Au})$ using an ion sputter coater. Finally, samples were viewed and imaged with a transmission electron microscope (TEM) (H-7700, Hitachi Company, Japan).

\section{Respiratory Control Ratio Detection}

Fresh pulmonary veins were quickly fixed with arsenate buffer. Freshly heart, liver, kidney, and intestine tissues were put into the separation buffer (sucrose $0.25 \mathrm{~mol} / \mathrm{L}, \mathrm{Na}_{2} \mathrm{EDTA} 0.1 \mathrm{mmol} / \mathrm{L}$, Tris0.01 mol/L) for homogenization. The homogenate was centrifuged $\left(4^{\circ} \mathrm{C}, 1,600 \mathrm{~g}, 12 \mathrm{~min}\right)$, and the supernatant was centrifuged $\left(4^{\circ} \mathrm{C}, 25,000 \mathrm{~g}, 15 \mathrm{~min}\right)$. The precipitate was collected and resuspended in $1 \mathrm{~mL}$ of the separation buffer. The measuring buffer (Tris $0.2 \mathrm{~mol} / \mathrm{L}, \mathrm{KCl} 15 \mathrm{mmol} / \mathrm{L}, \mathrm{KH}_{2} \mathrm{PO}_{4} 15 \mathrm{mmol} / \mathrm{L}$, $\mathrm{Na}_{2}$ EDTA $1 \mathrm{mmol} / \mathrm{L}, \mathrm{MgCl}_{2} 5 \mathrm{mmol} / \mathrm{L}$, sucrose $0.25 \mathrm{~mol} / \mathrm{L}$ ) was heated to $30^{\circ} \mathrm{C}$, and then added $0.2 \mathrm{~mL}$ of the mitochondrial mixture to the reaction chamber and equilibrated for $20 \mathrm{~s}$, and finally added $10 \mu \mathrm{L}$ sodium malate and $10 \mu \mathrm{L}$ sodium glutamate and $5 \mu \mathrm{L}$ ADP in sequence. The respiratory control ratio was measured by the mitochondrial dissolved oxygen meter (MT 200, Stranthkelvin, United Kingdom).

\section{Mitochondrial Morphology Observation by Immunofluorescence}

VECs were seeded in the confocal chamber and incubated with Mito-tracker $(1: 10,000)$ for $30 \mathrm{~min}$ at $37^{\circ} \mathrm{C}$. Mitochondrial morphology was visualized using confocal laser scanning microscopy (Leica SP5, Germany). The length of mitochondria was analyzed by Image J software ${ }^{1}$, which contains a Mitochondrial Network Analysis (MiNA) toolset (Valente et al., 2017).

\section{Prediction of ssKSRs With GPS}

The prediction of the Drp1 phosphorylation network and sitespecific kinase-substrate relations (ssKSRs) were conducted by a software package of iGPS (GPS algorithm with the interaction filter $)^{2}$. Protein kinases are classified into four levels, including group, family, subfamily, and single kinase. In total, GPS contains 144 and 69 individual predictors to predict ssKSRs from primary sequences of proteins for serine/threonine kinases (STKs) and tyrosine kinases (TKs), respectively (Wang et al., 2020).

\section{Statistical Analysis}

Statistical analyses were performed using SPSS 17.0 (SPSS Inc., Chicago, IL, United States). Data are presented as means \pm SD of at least three independent experiments. One-way analysis of variance and post hoc test (S-N-K/LSD) were used to analyze the difference between experimental groups. Survival time was analyzed by the median and interquartile range and KaplanMeier survival analyses and the log-rank test. Values of $p<0.05$ were considered statistically significant.

\footnotetext{
${ }^{1}$ https://fiji.sc/

${ }^{2}$ http://igps.biocuckoo.org/down.php
} 


\section{RESULTS}

\section{Protective Effects of Dexmedetomidine on the Vascular Permeability in Septic Rats}

In order to observe the effect of Dex on vascular endothelial barrier function, the permeability of lung and intestinal vascular to FITC-BSA or Evans blue following sepsis and the expression of intercellular junction proteins were measured. According to the guidelines for sepsis, after $12 \mathrm{~h}$ of CLP, rats received fluid resuscitation for $3 \mathrm{~h}$ with lactated Ringer's solution (LR) (35 $\mathrm{mL} / \mathrm{kg}$ ), vasopressor (dopamine $5 \sim 10 \mu \mathrm{g} / \mathrm{kg} / \mathrm{min}$ ), and antibiotic (cefuroxime sodium, $100 \mathrm{mg} / \mathrm{kg}$ ) as the conventional treatment (CT) (Allen et al., 2019). While rats in the Dex group received $10 \mu \mathrm{g} / \mathrm{kg}$ dexmedetomidine $30 \mathrm{~min}$ before and $12 \mathrm{~h}$ after CLP in addition to receiving $\mathrm{CT}$, and the sepsis group had no treatment. The results showed that the pulmonary vascular permeability of septic rats was significantly increased. It appeared the vascular leakage of FITC-BSA (yellow arrows) and Evans blue were increased by 124 and $148 \%$ as compared with the sham group $(P<0.01)$. CT did not effectively decrease the pulmonary vascular permeability $(P>0.05)$, while administration of Dex could significantly alleviate the leakage. Compared with the CT group, Evans Blue exudation in the Dex group was decreased by $30 \%(P<0.01)$ (Figure 1A), and the leakage of FITC-BSA was decreased by $34.5 \%(P<0.01)$ (Figures 1B,C).

To further explore the effect of Dex on vascular leakage after sepsis, the mesenteric venule branch was used as a microcirculation representative, the leakage of FITC-BSA from the mesenteric micro-vessels was directly measured through intravital microscopy. Following injecting FITC-BSA $(0,1,3,6 \mathrm{~min})$ intravenously, the change of FITC-BSA transmittances was observed. The results showed that the endothelial barrier function of mesenteric microvessels in septic rats was significantly impaired, and the exudation of FITC-BSA was notably increased. A large amount of FITC-BSA leaked out from the blood vessel after $6 \mathrm{~min}$ in septic rats $(P<0.01)$. CT did not improve the permeability of mesenteric microvessels effectively $(P>0.05)$ while Dex distinctly alleviated vascular leakage and the FITC-BSA permeability decreased by $39.5 \%(P$ $<0.01$ ) compared to the CT group (Figures 1D,E).

In general, cell connections such as tight junctions and adhesion junctions play an important role in the permeability of vascular endothelial cells. To investigate the effect of Dex on cell connections in pulmonary vascular endothelial, we observed the changes of tight junctions by the TEM. The results showed that the tight junctions between endothelial cells were closed and dense in the sham group. In the sepsis group, the vascular endothelial cells were swollen, and the tight junctions were obviously damaged, showing a state of relaxation and shedding. CT did not significantly improve the damaged tight junctions, while were restored to a close degree in the Dex group (Figure 1G). There are several key proteins participating in the regulation of vascular leakage, such as tight junction proteins (ZO-1, Occludin), and adherent junction protein (VEcadherin). The changes of ZO-1, Occludin, and VE-cadherin were further observed. The results showed that the expressions of ZO-1, Occludin, and VE-cadherin in septic rats were distinctly reduced $(P<0.01)$, and CT could not effectively change the decrease of intercellular junction proteins. Following Dex administration, the expressions of ZO-1, Occludin, and VEcadherin were evidently improved, increasing by $60.3,41.9$, and $70.6 \%$, respectively $(P<0.01)$ (Figure $1 \mathbf{F})$. These results indicate that Dex has a protective effect on the vascular endothelial barrier function of septic rats.

\section{Protective Effects of Dexmedetomidine on the Permeability of VECs After Sepsis}

To further investigating the effect of Dex in vitro, the permeability of VECs following LPS stimulation was measured. Cells were cultured till the confluence reached $60-70 \%$, and then LPS (1 $\mu \mathrm{g} / \mathrm{mL}$ ) was incubated for $12 \mathrm{~h}$ in the sepsis group. The Dex group was incubated with $0.1 \mu \mathrm{M}$ dexmedetomidine for $30 \mathrm{~min}$ before adding LPS $(1 \mu \mathrm{g} / \mathrm{mL})$. The results showed that the transmembrane electrical resistance (TER) of VEC decreased by $73.7 \%$ after LPS stimulation compared with the normal group. Meanwhile, the BSA leakage of VECs obviously increased $(P<0.01)$. Dex could notably improve the permeability of VECs. Compared with the LPS group, TER increased by $94.1 \%$, and the BSA leakage decreased by $8.9 \%$ in VECs of the Dex group $(P<0.01)$ (Figures 2A,B). Next, we observed the intercellular junction proteins expressions of VECs. The immunofluorescence results revealed that the ZO-1 was distributed continuously along the vascular endothelial cell membrane in the normal group, while in the LPS group, the fluorescence intensity of ZO-1 was weakly expressed and loosely distributed with gaps (Figure 2C), and the expressions of ZO-1, VE-cadherin, and Occludin, respectively, decreased by $74.3,74$, and $83.06 \%$ as compared to the normal group $(P<0.01)$ (Figures $2 D-G)$. Following Dex administration, the distribution of ZO-1 was improved with complete structure and clear boundary; simultaneously, in comparison with the LPS group, the expression of ZO-1, VEcadherin, and Occludin were increased by $172.8,200 \%(P<0.01)$, and $182.3 \%(P<0.05)$, respectively (Figures $2 \mathrm{D}-\mathbf{G})$. These results further suggest that Dex has a protective effect on the vascular endothelial barrier function of septic rats.

\section{Effects of Dexmedetomidine on Mitochondrial Fission and Mitochondrial Functions After Sepsis}

Mitochondria are dynamic organelles that undergo remodeling via fusion and fission. The fine balance between these two opposing processes determines mitochondrial morphometric properties and functions. Excessive mitochondrial fission leads to mitochondrial and organ dysfunction. Whether Dex exerts a protective effect on vascular endothelial barrier function by regulating mitochondrial fission is unclear. In the present study, we examined the morphologic changes of mitochondria in pulmonary veins with a TEM and found that the number of mitochondria substantially increased, and the aspect ratio of mitochondria decreased by $69.3 \%$ after sepsis $(P<0.01)$. The mitochondrial morphology did not improve, remaining 

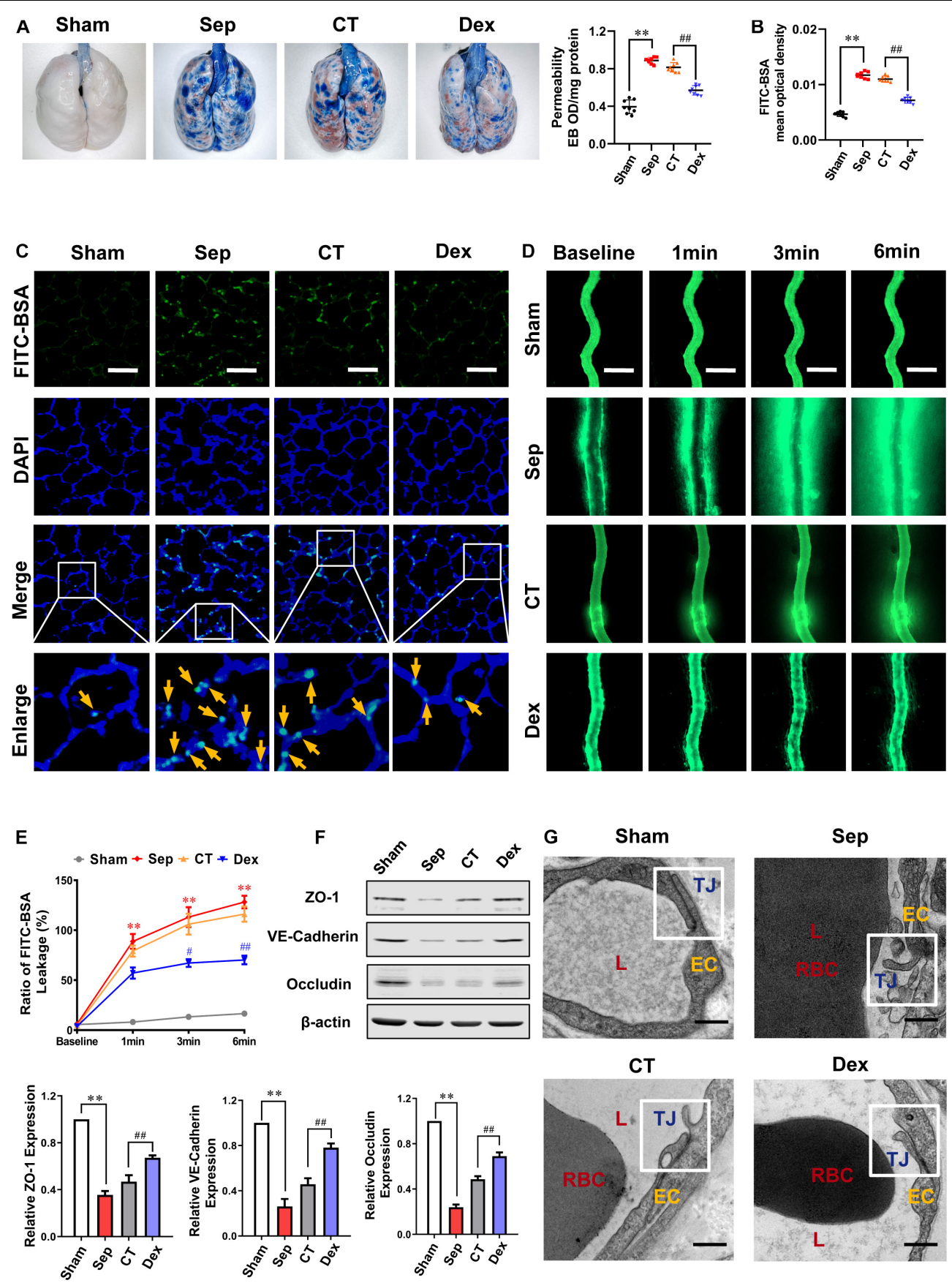

FIGURE 1 | Protective effect of dexmedetomidine on vascular permeability of septic rats. (A) Vascular permeability of the lung, measured by the leakage of Evans Blue, $n=8$. (B,C) Vascular permeability of the lung, measured by the mean optical density of intravenously injected FITC-BSA in vivo (Bar, $50 \mu \mathrm{m}), n=8$. (D,E) The FITC-BSA leakage of mesentery microvessels in rats, dynamically measured by intravital microscopy in vivo (Bar, $100 \mu \mathrm{m}), n=8$. (F) Western blot analysis of ZO-1, Occludin and VE-cadherin in the superior mesenteric vein of rats treated with CLP, $n=3$. (G) Representative transmission electron microscope microphotographs of tight junctions of pulmonary venules (Bar, $200 \mathrm{~nm}$ ), $n=8$. EC, endothelial cell; RBC, red blood cell; TJ, tight junction; L, lumen; Sham, sham group; Sep, sepsis group; CT, conventional treatment group; Dex, dexmedetomidine group. ${ }^{* *} P<0.01$, as compared with sham group; ${ }^{\#} P<0.01$, as compared with $\mathrm{CT}$ group; ${ }^{\#} P<$ 0.05, as compared with CT group.

short and small $(P>0.05)$ after CT. In contrast, the mitochondrial morphology was significantly restored, and the aspect ratio increased by $47.7 \%$ in the Dex group $(P<0.01)$ (Figure 3A). At the cellular level, the morphological changes of mitochondria were further observed by a laser confocal microscope. Each group randomly selected 50 cells and the mitochondrial morphology was blindly scored and classified into three categories: Long $(>6 \mu \mathrm{m})$, Middle $(3-6 \mu \mathrm{m})$, 

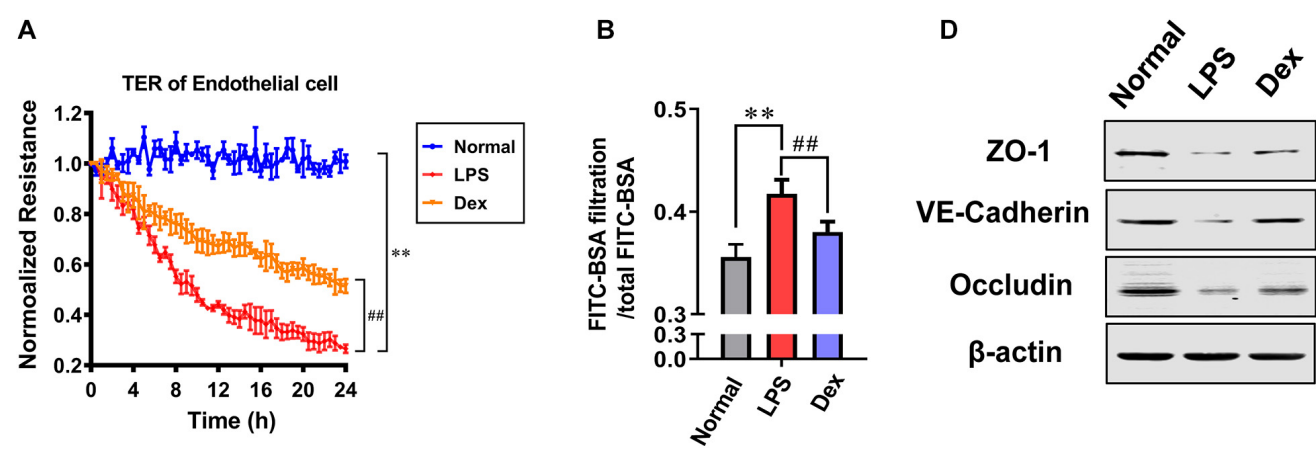

C

Tight junctions in VECs
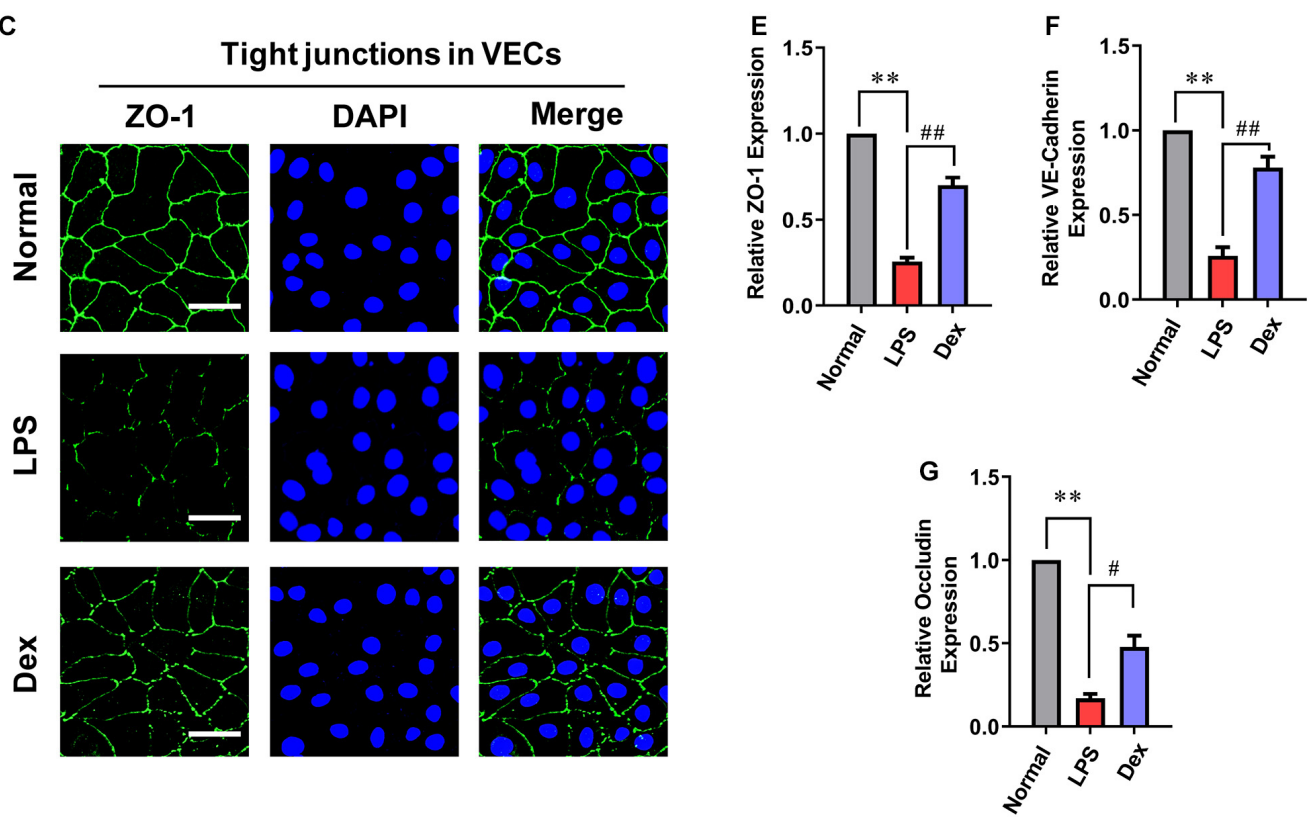

FIGURE 2 | The influence of dexmedetomidine on the permeability of vascular endothelial cells after sepsis. (A) Effects of dexmedetomidine on the TER (transendothelial electrical resistance) of VEC monolayers after sepsis, $n=3$. (B) Effects of dexmedetomidine on the infiltration rate of FITC-BSA in monolayer VECs after sepsis, $n=3$. (C) Measurement of the expression of ZO-1(green) after sepsis in VECs by immunofluorescence (Bar, $25 \mu \mathrm{m}$ ), $n=3$. (D-G) Western blot analysis of ZO-1, Occludin and VE-cadherin in VECs after sepsis, $n=3$. Normal: nomal group; LPS: Ips group; Dex: dexmedetomidine group. ** $P$ < 0.01 , as compared with normal group; ${ }^{\#} P<0.01$, as compared with Ips group; ${ }^{\#} P<0.05$, as compared with Ips group.

Short ( $<3 \mu \mathrm{m})$ (Gao et al., 2017). The results showed that the mitochondria were mostly in elongated shapes under normal conditions, of which 54\% were long mitochondria, $40 \%$ were medium mitochondria, and only $6 \%$ were short. However, the proportion of short mitochondria distinctly increased and reached $66 \%$ after LPS stimulation. Dex treatment could recover the long mitochondria accounted for $37 \%$ and the proportion of short reduced to $26 \%$ (Figure 3B), indicating that Dex could effectively alleviate mitochondrial fission. Since mitochondrial morphology is subject to changes in the frequency of division and fusion, we further continuously observed $10 \mathrm{~min}$ to record the frequency of mitochondrial divisions by confocal immunostaining, and it was found that the frequency of mitochondrial divisions increased by $121 \%$ after sepsis $(P<0.01)$, while compared with the LPS group, the frequency of mitochondrial divisions after Dex treatment decreased by $33.8 \%$ $(P<0.01)$ (Figures 3C,D).

Next, we explored whether Dex can protect mitochondrial function by protecting mitochondrial morphology. Mitochondrial membrane potential $(\Delta \Psi \mathrm{m})$ is a vital part that directly affects the mitochondrial capacity, and the generation of ROS is closely related to the functional status of mitochondria. Therefore, we observed $\Delta \Psi \mathrm{m}, \mathrm{ROS}$, and ATP production of VECs. The results showed that compared with the normal group, $\Delta \Psi \mathrm{m}$ and ATP levels were significantly reduced, and the generation of ROS was increased after $12 \mathrm{~h}$ LPS treatment $(P<0.01)$. Meanwhile, $\Delta \Psi \mathrm{m}$ and ATP levels in the Dex group, respectively, increased by 121.7 and $64.7 \%$, and ROS decreased by $40 \%(P<0.01)$ in comparison with the LPS group (Figures $3 \mathrm{E}-\mathbf{H}$ ). In addition to VECs, Dex also 


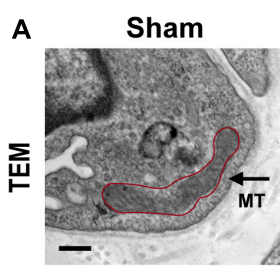

B Normal
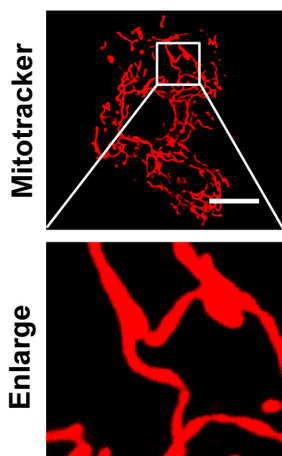

D Recording

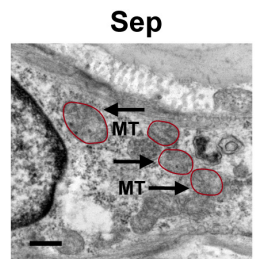

LPS
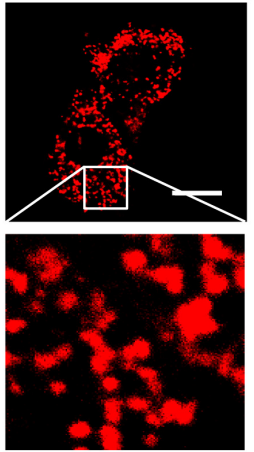

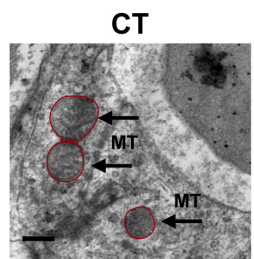

Dex
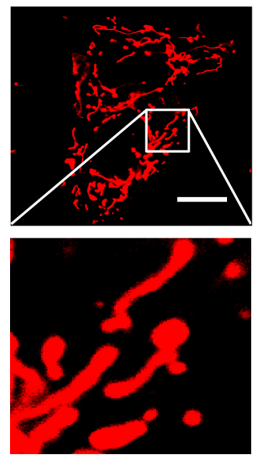
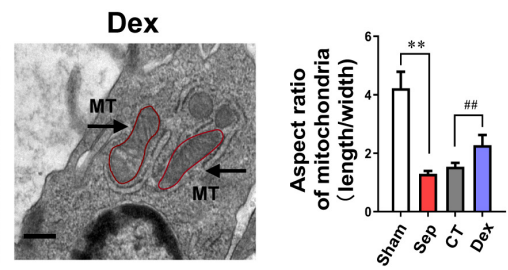

C
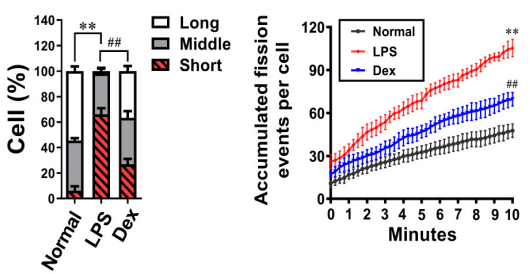
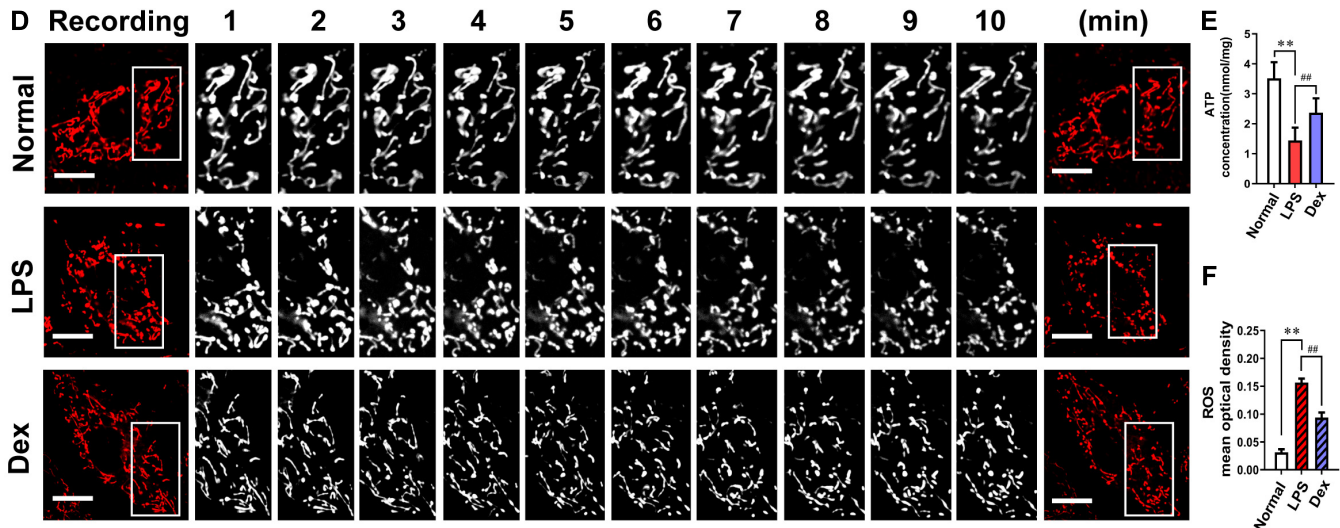

$\Delta \Psi \mathrm{m}$
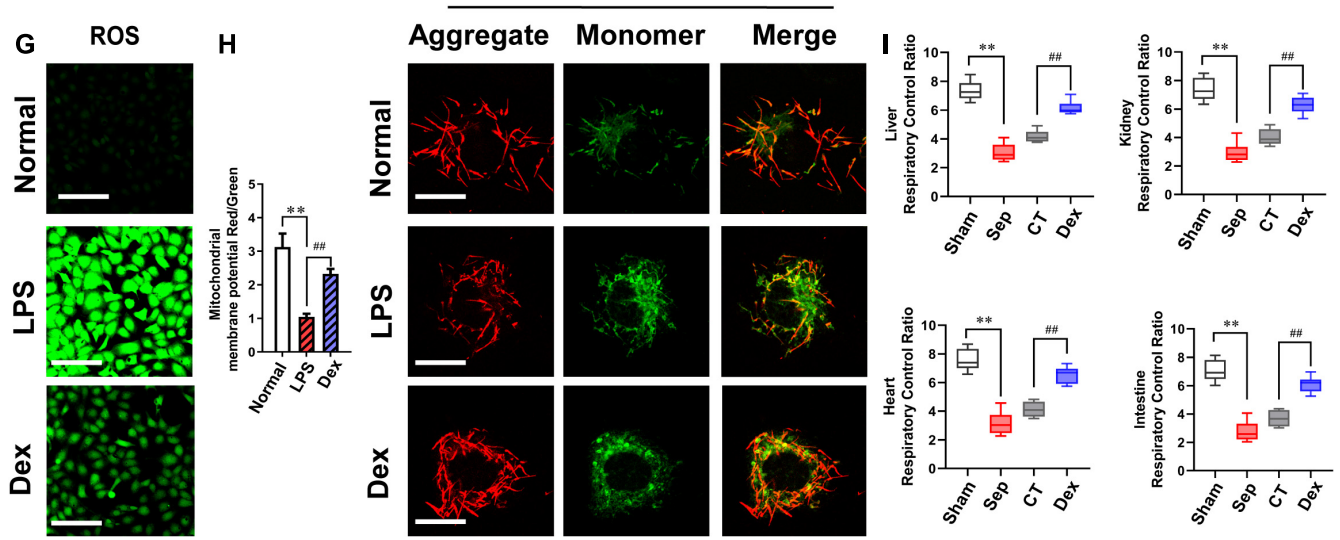

FIGURE 3 | The effects of dexmedetomidine on mitochondrial fission and mitochondrial functions after sepsis. (A) TEM (transmission electronic microscopy) images to observe mitochondrial morphology of pulmonary venules in septic rats (Bar, $200 \mathrm{~nm}), n=8$. (B) Confocal images to observe mitochondrial morphology of VECs after sepsis (Bar, $25 \mu \mathrm{m}), n=50$. (C,D) Time-lapse images of mitochondrial morphologic alternation of VECs per $15 \mathrm{~s}$ after sepsis by confocal immunostaining (Bar, $20 \mu \mathrm{m}), n=3$. (E) Effects of dexmedetomidine on the ATP of VECs after sepsis, $n=3$. (F-H) Representative confocal images of ROS(Bar, $100 \mu \mathrm{m})$ and $\Delta \Psi \mathrm{m}$ (Bar, $25 \mu \mathrm{m}$ ) after sepsis in VECs, $n=3$. (I) Effects of dexmedetomidine on the respiratory control ratio in septic rats, $n=3$. Sham: sham group; Sep: sepsis group; CT: conventional treatment group; Normal: nomal group; LPS: Ips group; Dex: dexmedetomidine group. ${ }^{* *} P<0.01$, as compared with normal group; ${ }^{\# \#} P<0.01$, as compared with Ips group. 
protected the mitochondrial functions of heart, liver, kidney, and intestinal mucosal epithelial cells in vivo. The respiratory control rates (RCRs) of liver, kidney, heart, and intestine tissues were distinctly reduced, and all of the reduction rates were higher than 55\% $(P<0.01)$. RCRs were not improved significantly after CT $(P>0.05)$, while Dex could effectively restore the above parameters. Compared with the CT group, RCRs of liver, kidney, heart, and intestine tissues increased by 47.4, 55.8, 58.7, and $66.7 \%$, respectively $(P<0.01)$ (Figure 3I). These results suggest that Dex can protect the vascular endothelial barrier function by alleviating excessive mitochondrial division and protecting mitochondrial function.

\section{Dexmedetomidine Reduced ER-MITO Contacts via Actin Polymerization Inhibition and Thus Inhibited Mitochondrial Fission}

Previous studies demonstrated (Wang et al., 2002; Wu et al., 2016; Chakrabarti et al., 2018) that endoplasmic reticulum and mitochondria (ER-MITO) contact played a vital role in mitochondrial fission by inducing mitochondrial pre-contraction before mitochondrial fission. However, whether Dex's regulation of mitochondrial division is related to ER-MITO contact has never been shown. Firstly, we used immunofluorescence to observe the effect of Dex on ER-MITO contact. The results found that LPS stimulation induced mitochondria to split, and the ER-MITO contact sites were significantly increased with evident mitochondrial pre-constriction traces compared with the normal group. After the treatment with Dex, the number of ER-MITO contact sites and mitochondrial pre-constriction were both reduced distinctly compared with the LPS group, showing a similar trend as the inhibition of mitochondrial division (Figure 4A).

The process of actin accumulated to bundles (F-actin) and pull the endoplasmic reticulum and result in ER-MITO contact was a vital initiation step in the mitochondrial preconstriction. To explore whether the increase in ER-MITO contact caused by Dex is related to F-actin, we further observed the effect of Dex on actin polymerization. The results showed that compared with the normal group, F-actin stress fibers increased notably after the stimulation of LPS and aggregated to form bundles (white arrows) in VECs, while Dex could inhibit actin aggregation from forming stress fibers (Figure 4B). The relationship between actin aggregation and mitochondrial division was further observed with a laser confocal microscope. The contacts between F-actin and mitochondria increased about 3-4 times after LPS stimulation as compared with the normal group $(P<0.01)$ (white circles). Meanwhile, short mitochondria increased from $7 \%$ in the normal group to $64 \%$ in the LPS group. In the Dex group, the contacts between F-actin and mitochondria were evidently reduced, and short mitochondria decreased to 26\% $(P<0.01)$ (Figures 4C-E).

Since Dex is a highly selective $\alpha_{2}$ adrenergic receptor agonist, we used the targeted blocker atipamezole (Ati) to illustrate whether Dex exerted the above effects through regulating $\alpha_{2}$ receptors. $1 \mu \mathrm{M}$ atipamezole was added to incubate the cells
10 min before adding $0.1 \mu \mathrm{M}$ dexmedetomidine, and the results were consistent with expectations. Ati could significantly antagonize the polymerization of actin caused by Dex and the increase of ER-MITO contact sites, thereby antagonizing the protective effect of Dex on excessive mitochondrial division (Figures 4A-E). These results suggested that Dex exerted the protective role in mitochondrial function by inhibiting actin polymerization via $\alpha_{2}$ receptors activation, reducing the number of ER-MITO contact sites, and suppressing mitochondrial division.

\section{Dexmedetomidine Phosphorylates ERK1/2-Mediated Mitochondrial Fission}

Based on the pre-constriction of mitochondria caused by ERMITO contact, Dynamin-related protein 1 (Drp1) is recruited to mitochondria and then cut mitochondria through GTPase, which is a vital link that leads to mitochondrial fission. As a member of the dynamin superfamily, Drp1 has many phosphorylation sites with various roles. The Ser 616 and Ser637 sites are critical for the translocation of Drp1 from the cytoplasm to mitochondria. Western Blotting results showed that there was no obvious difference in total expression of Drp1 after LPS stimulation compared to the normal group while the phosphorylation of Drp1 Ser616 increased by $238.3 \%$ $(P<0.01)$, and the phosphorylation of Ser637 decreased by $57 \%(P<0.01)$. Moreover, the expression of Drp1 increased in mitochondria and decreased in cytoplasm significantly after sepsis $(P<0.01)$ (Figures 5A,B), and the Adenine nucleotide translocase (ANT) was used as a reference when detecting the change of Mito-Drp1. The confocal images indicated that the co-localization ratio between Drp1 and mitochondria distinctly increased, and the length of mitochondria shortened from $16.10 \pm 5.43 \mu \mathrm{m}$ in the normal group to $5.47 \pm 2.91 \mu \mathrm{m}$ after sepsis $(P<0.01)$ (Figures 5C-E). Compared with the LPS group, the phosphorylation level of Ser616 reduced by $43.5 \%$, and the level of Ser637 increased by $61.1 \%$. The mitochondrial translocation of Drp1 reduced, and the expression of Drp1 in cytoplasm notably increased in the Dex group $(P<0.01)$ (Figures 5A,B). Furthermore, the co-localization ratio reduced, and the mitochondrial length was $10.86 \pm 5.91 \mu \mathrm{m}$ in the Dex group $(P<0.01)$ (Figures $\mathbf{5 C}-\mathbf{E})$. We further observed the effect of Dex on mitochondrial fusion proteins and found no significant differences in the expressions of OPA1, Mfn1, and Mfn2 following Dex administration (Supplementary Figure 1). Overall, these results suggested that Dex could effectively inhibit the activation and mitochondrial translocation of Drp1, and influence the morphology and function of mitochondria.

To further explore the mechanism of Dex regulating drp1 activation, a software package in vivo GPS (iGPS 1.0) (Wang et al., 2020) was used to predict the site-specific kinase for all phosphorylation-sites of Drp1. The prediction results showed that the extracellular regulated protein kinases1/2 (ERK1/2) could significantly regulate the Ser616 site of Drp1 (Figure 5F and Table 1), and previous studies (Zhao et al., 2019; Zhang et al., 2019) also reported that ERK1/2 is an essential kinase that regulates Drp1. So the effect of ERK1/2 on Dex regulating 


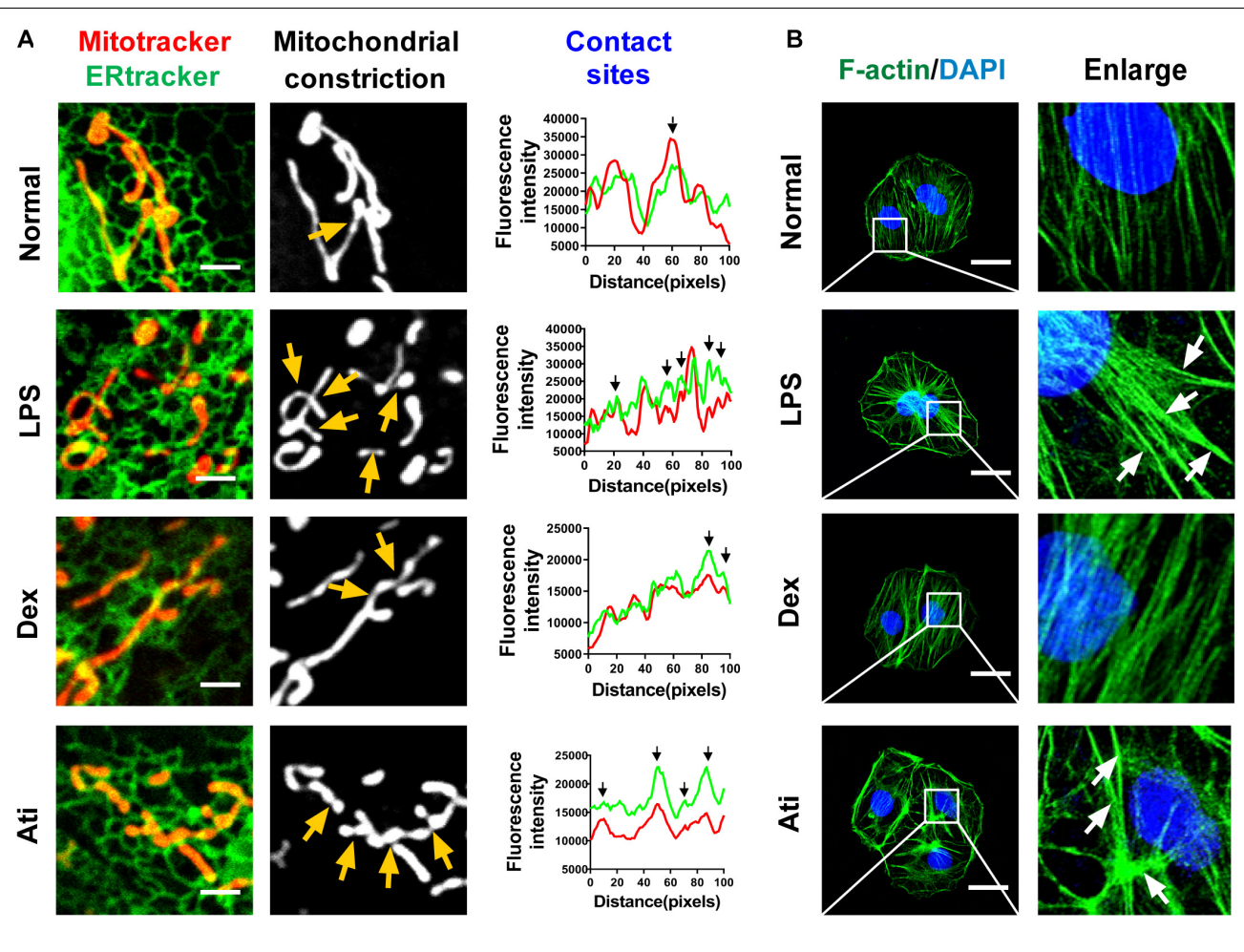

C
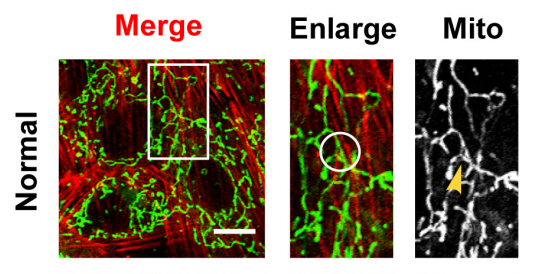

F-actin

D
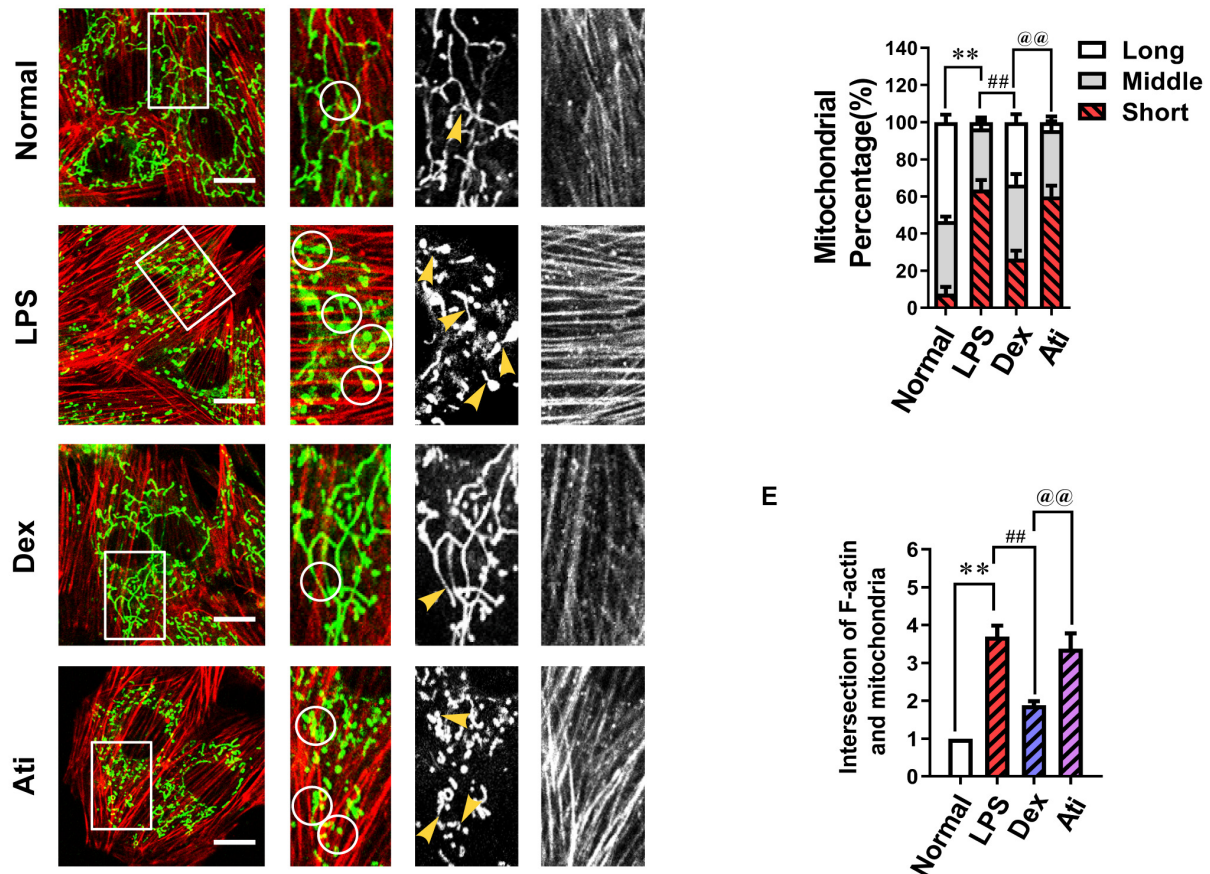

E

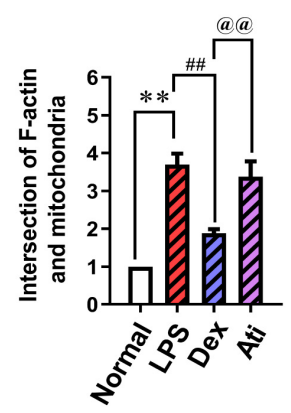

FIGURE 4 | The effects of dexmedetomidine on F-actin and ER-MITO contact of vascular endothelial cells after sepsis. (A) Representative fluorescent images of ER-MITO contact transformed with ERtracker and Mitotracker in VECs after sepsis (Bar, $5 \mu \mathrm{m}$ ), $n=3$. The sites of mitochondrial fission (yellow arrows) correspond to black arrows of contact sites on the line scan. (B) Confocal images to observe the effect of dexmedetomidine on the polymerization of actin (Bar, $25 \mu \mathrm{m}$ ), $n=3$. (C) Confocal images to observe mitochondria and F-actin of VECs after sepsis(Bar, $20 \mu \mathrm{m}), n=3$. (D) The statistical analysis of mitochondrial morphology of VECs in different groups. Quantitation was performed in triplicate and scored into three categories: long, middle, and short mitochondria, with 50 cells scored per group. (E) The intersections of F-actin and mitochondria of VECs in different groups were calculated by Image $\mathrm{J}$ software. N, nomal group; LPS, Ips group; Dex, dexmedetomidine group; Ati, Atipamezole group. ${ }^{* *} P<0.01$, as compared with normal group; ${ }^{\# \#} P<0.01$, as compared with lps group; ${ }^{\circledR} P<0.01$, as compared with dex group. 

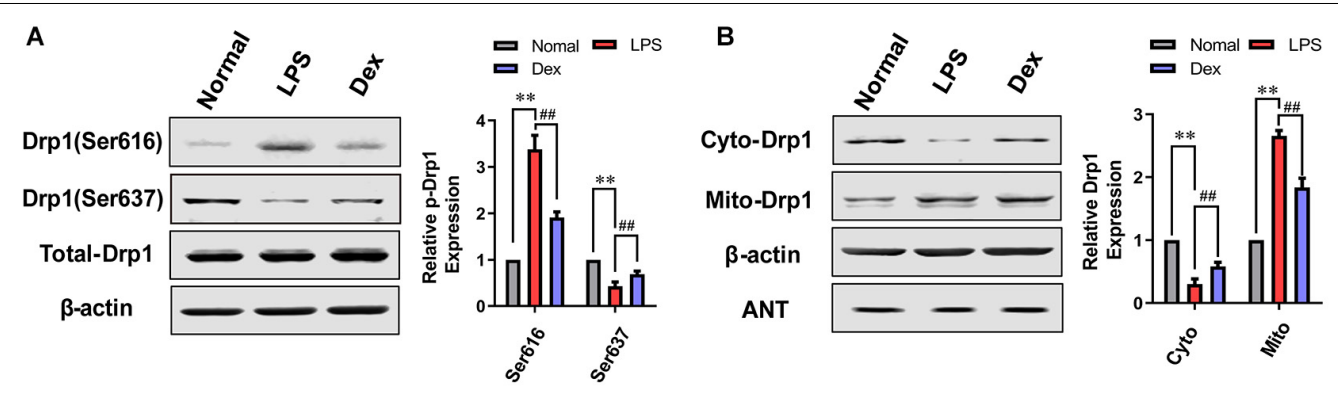
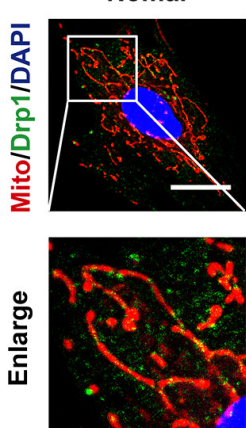

G
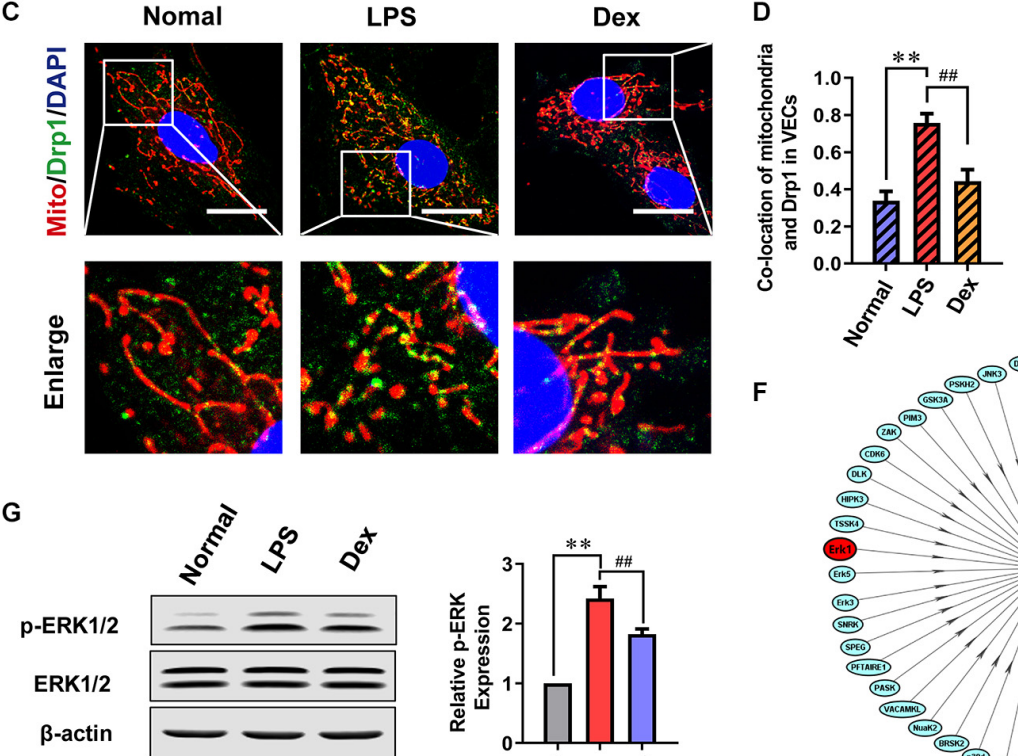

E

$\mathbf{F}$

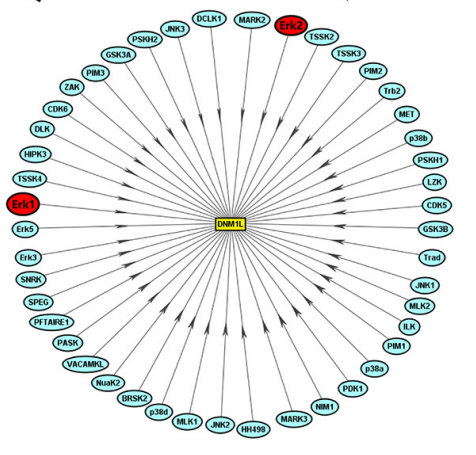

H
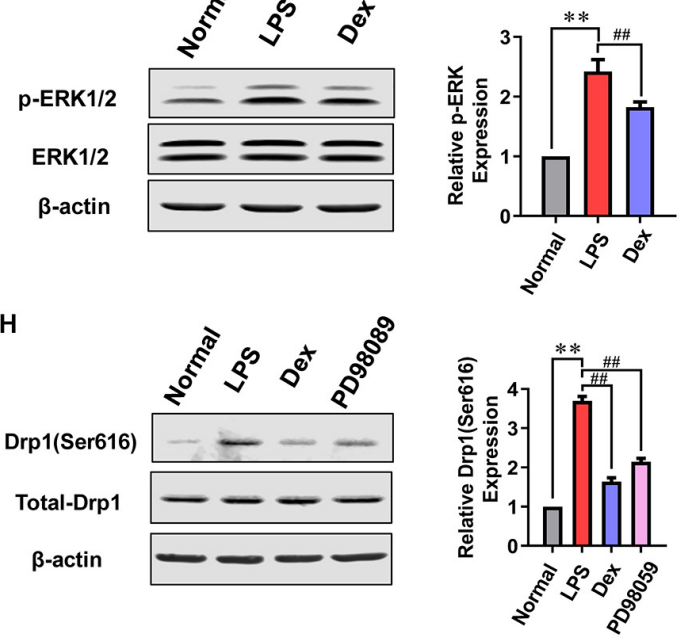

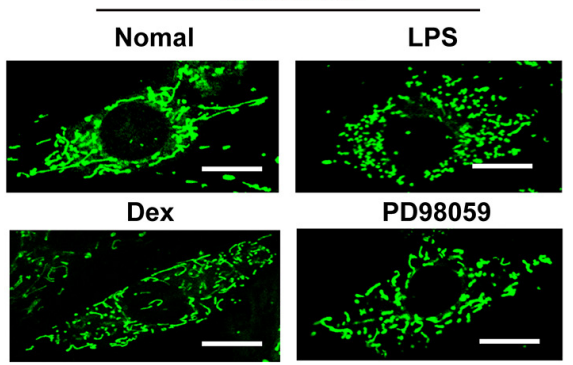

$J$

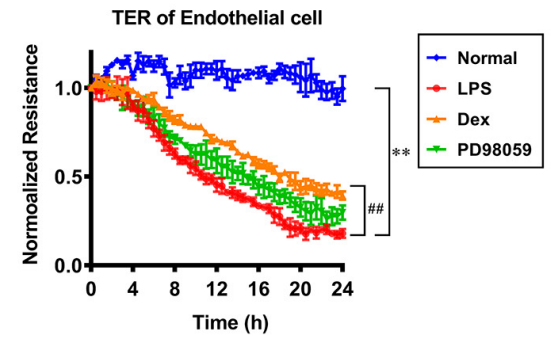

K

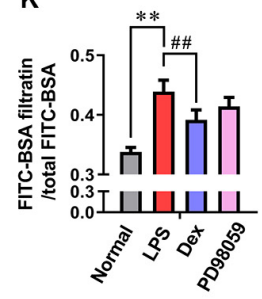

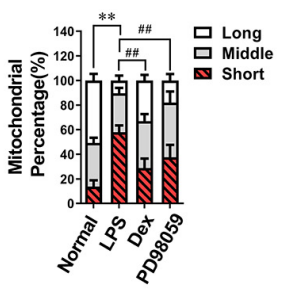

FIGURE 5 | Dexmedetomidine regulates ERK1/2-mediated mitochondrial fission. (A) Effects of dexmedetomidine on the phosphorylation of Drp1 after sepsis in VECs detected by Western Blotting, $n=3$. (B) Effects of dexmedetomidine on the mitochondrial translocation of Drp1 after sepsis in VECs, $n=3$. (C,D) The co-location of Drp1 and mitochondria of VECs in each group (Bar, $25 \mu \mathrm{m}), n=3$. (E) The length of mitochondria of VECs in each group. (F) Drp1 protein phosphorylation network (PPN) predicted by iGPS 1.0 software. (G) Effects of dexmedetomidine on the phosphorylation of ERK1/2 after sepsis in VECs, $n=3$. (H) Effects of dexmedetomidine and ERK1/2 inhibitor on the phosphorylation of ERK1/2 after sepsis in VECs, $n=3$. (I) Mitochondrial morphology in

PD98059-treated VECs after sepsis (Bar, $15 \mu \mathrm{m}), n=3$. (J,K) Effects of PD98059 on the TER and FITC-BSA infiltration rate of VEC monolayers after sepsis, $n=3$. Normal, normal group; LPS, Ips group; Dex, dexmedetomidine group. PD98059: ERK1/2 inhibitor group. ${ }^{* *} P<0.01$, as compared with normal group; \#\# $P<0.01$, as compared with Ips group. 
TABLE 1 | The prediction outcome of Drp1 ser616 and ser637 phosphorylation sites and their site-specific kinase.

\begin{tabular}{|c|c|c|c|c|c|c|c|c|}
\hline ID & Position & Code & Peptide & Gene name & Kinase name & Interaction & Predictor & Score \\
\hline sp| 000429 & 616 & $S$ & PIPIMPASPQKGHAV & DNM1L & CDK5 & String & CMGC/CDK & 2.61 \\
\hline sp| O00429 & 616 & S & PIPIMPASPQKGHAV & DNM1L & CDK6 & String & CMGC/CDK & 2.61 \\
\hline sp| 000429 & 616 & S & PIPIMPASPQKGHAV & DNM1L & PFTAIRE1 & String & CMGC/CDK & 2.61 \\
\hline sp| O00429 & 616 & S & PIPIMPASPQKGHAV & DNM1L & HIPK3 & String & CMGC/DYRK & 2.33 \\
\hline sp| 000429 & 616 & S & PIPIMPASPQKGHAV & DNM1L & Erk1 & String & CMGC/MAPK & 3.47 \\
\hline sp| 000429 & 616 & $S$ & PIPIMPASPQKGHAV & DNM1L & Erk5 & String & CMGC/MAPK & 3.47 \\
\hline sp| 000429 & 616 & S & PIPIMPASPQKGHAV & DNM1L & Erk3 & String & CMGC/MAPK & 3.47 \\
\hline sp| 000429 & 616 & S & PIPIMPASPQKGHAV & DNM1L & JNK3 & String & CMGC/MAPK & 3.47 \\
\hline sp| 000429 & 616 & S & PIPIMPASPQKGHAV & DNM1L & JNK1 & String & CMGC/MAPK & 3.47 \\
\hline sp| 000429 & 616 & S & PIPIMPASPQKGHAV & DNM1L & p38b & String & CMGC/MAPK & 3.47 \\
\hline sp| 000429 & 616 & S & PIPIMPASPQKGHAV & DNM1L & JNK2 & String & CMGC/MAPK & 3.47 \\
\hline sp| 000429 & 616 & S & PIPIMPASPQKGHAV & DNM1L & Erk2 & String & CMGC/MAPK & 3.47 \\
\hline sp| 000429 & 616 & S & PIPIMPASPQKGHAV & DNM1L & p38a & String & CMGC/MAPK & 3.47 \\
\hline sp| 000429 & 616 & S & PIPIMPASPQKGHAV & DNM1L & p38d & String & CMGC/MAPK & 3.47 \\
\hline sp| 000429 & 637 & S & VPVARKLSAREQRDC & DNM1L & SPEG & String & CAMK & 2.86 \\
\hline sp| 000429 & 637 & S & VPVARKLSAREQRDC & DNM1L & TSSK4 & String & CAMK & 2.86 \\
\hline sp| 000429 & 637 & S & VPVARKLSAREQRDC & DNM1L & DCLK1 & String & CAMK & 2.86 \\
\hline sp| 000429 & 637 & S & VPVARKLSAREQRDC & DNM1L & PSKH2 & String & CAMK & 2.86 \\
\hline sp| 000429 & 637 & S & VPVARKLSAREQRDC & DNM1L & Trb2 & String & CAMK & 2.86 \\
\hline sp| 000429 & 637 & S & VPVARKLSAREQRDC & DNM1L & PIM3 & String & CAMK & 2.86 \\
\hline sp| 000429 & 637 & S & VPVARKLSAREQRDC & DNM1L & Trad & String & CAMK & 2.86 \\
\hline sp| 000429 & 637 & S & VPVARKLSAREQRDC & DNM1L & PSKH1 & String & CAMK & 2.86 \\
\hline sp| 000429 & 637 & S & VPVARKLSAREQRDC & DNM1L & TSSK3 & String & CAMK & 2.86 \\
\hline sp| 000429 & 637 & S & VPVARKLSAREQRDC & DNM1L & TSSK2 & String & CAMK & 2.86 \\
\hline sp| 000429 & 637 & $S$ & VPVARKLSAREQRDC & DNM1L & VACAMKL & String & CAMK & 2.86 \\
\hline sp| 000429 & 637 & S & VPVARKLSAREQRDC & DNM1L & PIM2 & String & CAMK & 2.86 \\
\hline sp| 000429 & 637 & $S$ & VPVARKLSAREQRDC & DNM1L & PIM1 & String & CAMK & 2.86 \\
\hline
\end{tabular}

Drp1 Ser 616 phosphorylation was further studied. The results pointed out that the phosphorylation of ERK1/2 increased by $142.4 \%$ in the LPS group $(P<0.01)$, and Dex could prominently reduce the ERK1/2 phosphorylation $(P<0.01)$ (Figure 5G). Besides, the ERK1/2 inhibitor (PD98059) $(10 \mu \mathrm{M}$ PD98059 was added to incubate the cells 10 min before adding $1 \mu \mathrm{g} / \mathrm{mL}$ LPS) could effectively inhibit the phosphorylation of Drp1 Ser616 (Figure 5H). Furthermore, PD98059 also could inhibit mitochondrial fission $(P<0.01)$ and slightly inhibit vascular leakage $(P>0.05)$ (Figures 5I-K). These results indicate that Dex could reduce mitochondrial division via regulating the activation and translocation of Drp1 by inhibiting the phosphorylation of ERK1/2.

\section{Effects of Dexmedetomidine on Survival Time, Survival Rate, and Organ Functions in Septic Rats}

To evaluate whether Dex can protect organ functions and prolong the survival time of septic rats by alleviating the vascular leakage, we detected the arterial blood gas and the level of CK$\mathrm{MB}, \mathrm{ALT}$, and Crea to reflect the degree of organ damage. We discovered that Dex could remarkably improve the functions of lung, heart, liver, and kidney in septic rats. Compared with the CT group, the $\mathrm{pH}$ value was significantly enhanced, and acidosis was alleviated after Dex treatment. Besides, $\mathrm{PaO}_{2}$ and
$\mathrm{PaCO}_{2}$ increased by 4.49 and $3.74 \%$, respectively $(P<0.05)$, while CK-MB, ALT, Crea were decreased by $13.1,25.4$, and $24.7 \%$ individually $(P<0.01)$ (Figures 6A-F).

Results showed that the $24 \mathrm{~h}$ survival rate of septic rats was only $6.25 \%(P<0.01)$, and CT slightly increased the survival rate and survival time compared with the sepsis group. While Dex could significantly improve the survival rate and survival time of septic rats compared with the CT group. The $24 \mathrm{~h}$ survival rate was $50 \%$, and the mean survival time was $23 \pm 13.25 \mathrm{~h}$ in the Dex group $(P<0.01)$ (Figure $6 \mathbf{G})$. In addition, we also verified the mechanism of Dex regulating ERK1/2 mediated Drp1 activation in vivo, and the results were consistent with those in vitro (Figure 6H). The results suggested that Dex could protect organ functions and prolong survival by inhibiting vascular leakage.

\section{DISCUSSION}

The vascular endothelial barrier dysfunction is the primary cause of sepsis-induced organ dysfunction (Armstrong et al., 2013; Vincent et al., 2013; Miranda et al., 2015; Schmidt et al., 2015). Previous studies found that dexmedetomidine protected against vital organ injury in sepsis by inhibiting inflammatory factors and reducing oxidative stress (Sun et al., 2019; Zi et al., 2019). The present study demonstrated that Dex could effectively alleviate sepsis-induced vascular leakage and vascular endothelial barrier 

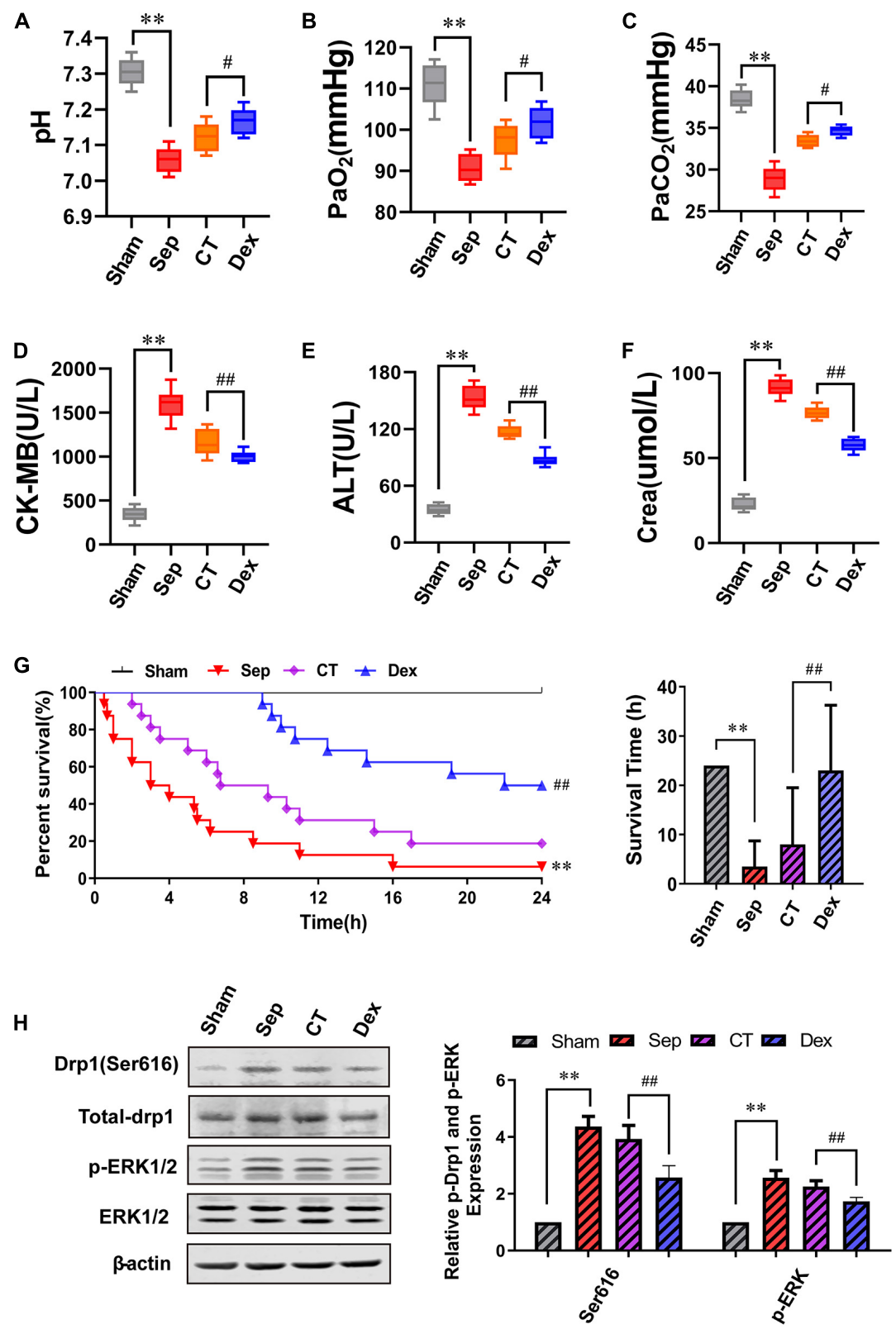

FIGURE 6 | The influence of dexmedetomidine on the survival rate, survival time and organ function of septic rats. (A-F) Statistical histogram of pH, $\mathrm{PaO}_{2}, \mathrm{PaCO}_{2}$, ALT, CK-MB, and Crea levels in each group, $n=8$. (G) Survival rate and survival time, $n=16$. (H) Effects of dexmedetomidine on the phosphorylation 616 site of Drp1 and ERK1/2 in septic rats detected by Western Blotting, $n=3$. Sham, sham group; Sep, sepsis group; CT, conventional treatment group; Dex. dexmedetomidine group. ${ }^{* *} P<0.01$, as compared with sham group; ${ }^{\#} P<0.01$, as compared with CT group; ${ }^{\#} P<0.05$, as compared with CT group.

dysfunction in septic rats, thus protect organ functions and ultimately prolong the survival rate and survival time of septic rats, which provided a novel sight for the treatment of sepsis with anesthetic drugs.

Recent studies pointed out that the protective effect of Dex on organ functions may closely related to the regulation of mitochondrial function. At present, it is believed that the main mechanisms to improve mitochondrial function are to activate the mitochondrial ATP-sensitive potassium channel and increase mitochondrial membrane potential (Yuan et al., 2017).
This study showed that Dex could suppress the permeability of VECs by protecting mitochondrial morphology. After sepsis, the mitochondrial morphology of VECs is damaged, and mitochondria divided excessively, accompanying ATP production obstacles, ROS production increases, mitochondrial membrane potential, and RCR decreases. However, Dex distinctly inhibited the mitochondrial division of VECs, improved mitochondrial morphology, and protected mitochondrial function. Generally, mitochondria are mostly in elongated or thread shapes in normal condition, while the pathological 


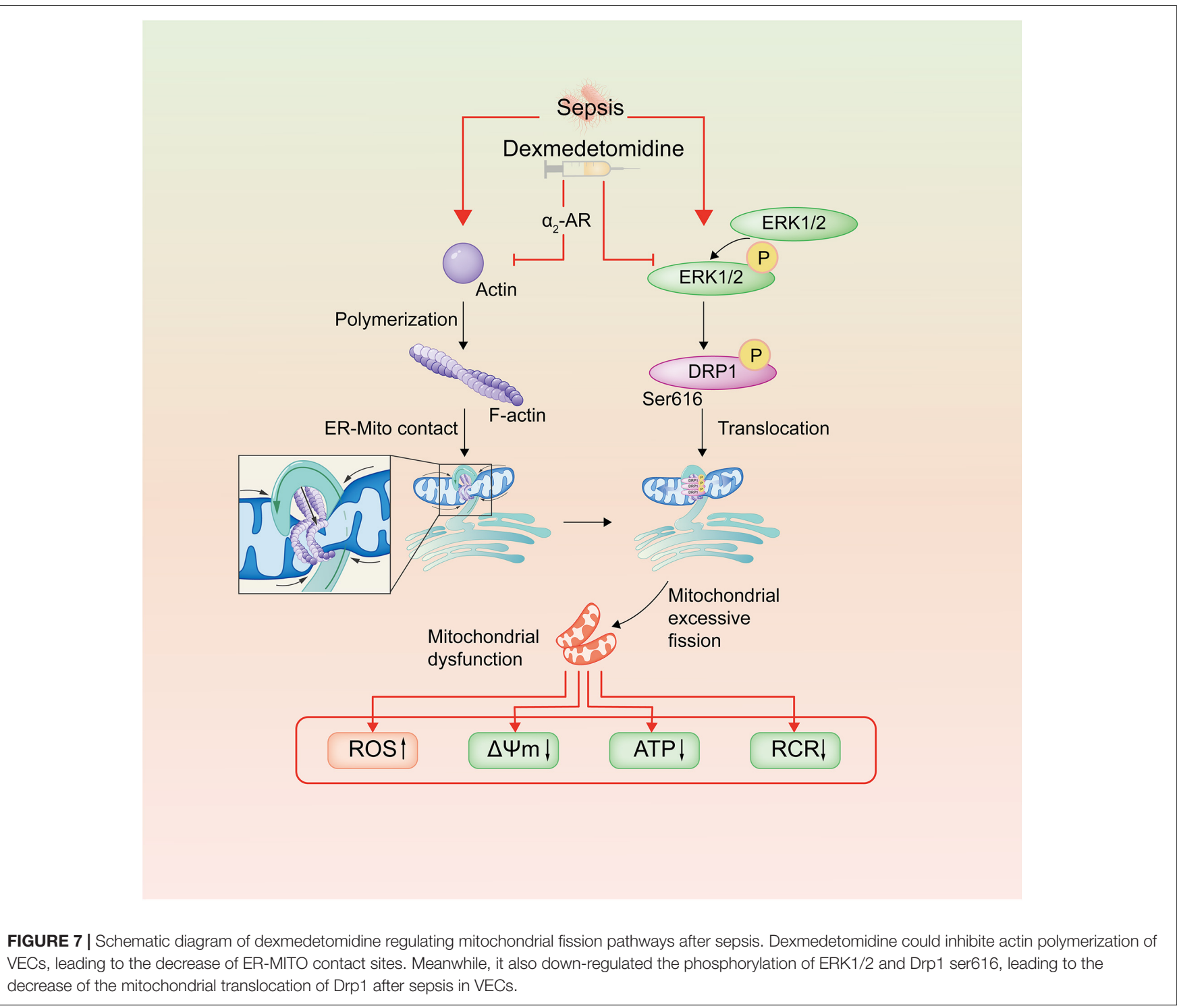

stimulation lead to fragmentation, furthermore, if the stimuli continue to exist, mitochondria will divide excessively, resulting in reduced ATP production, disturbance of calcium metabolism, and finally develop into mitochondria dysfunction. Numerous studies have confirmed that mitochondrial dysfunction plays a vital role in MODS, and the dysfunction degree is directly related to the outcome of patients (Brooks et al., 2009; Eismann et al., 2009; Liu et al., 2012; Duan et al., 2020). Our study demonstrated that Dex could protect mitochondrial function by improving mitochondrial morphology.

The key links of mitochondrial fission included ERMITO contact-induced mitochondrial pre-constriction and Drp1-related mitochondria split. Previous studies demonstrated that ER-MITO contact was a functional contact structure formed by the formation of a complex between the endoplasmic reticulum and mitochondria such as IP3R-GRP75-VDAC complex and so on (Friedman et al., 2011). Early reports revealed that the primary function of ER-MITO contact was to regulate the transfer of calcium and lipids between the endoplasmic reticulum and mitochondria. In recent years, researchers have found that ER-MITO contact could participate in various diseases by regulating mitochondrial structure (Rowland and Vorletz, 2012). The studies showed that actin polymerization could increase the formation of ER-MITO contacts (Wales et al., 2016) and promote mitochondrial fission under the effect of Myosin Light Chain Kinase (MLCK) or ROCK. In the present study, we demonstrated that actin in VECs polymerized to form stress fibers after stimulation with LPS for $12 \mathrm{~h}$, which increased ER-MITO contact sites and mitochondrial fission. While Dex could inhibit the aggregation of actin and reduce ER-MITO contact sites, leading to the inhibition of mitochondrial fission. Meanwhile, we also found that atipamezole, the highly selective $\alpha_{2}$ receptor blocker, could significantly antagonize the effects of Dex, suggesting that Dex may inhibit actin polymerization of VECs after sepsis by activating $\alpha_{2}$ receptors. Furthermore, studies (Hatch et al., 2014) have found that actin polymerization 
can activate the ER-localized inverted formin 2 (INF2), and the activated INF2 aggregated between the mitochondria and the ER, which further increased the formation of ER-MITO contacts. However, whether Dex exerts the same modulation effect on INF2 needs further investigation. Previous studies have shown that increased intracellular $\mathrm{Ca}^{2+}$ influx can activate MLCK by mediating $\mathrm{Ca}^{2+} / \mathrm{CaM}-\mathrm{PKC}$ and $\mathrm{Ca}^{2+}$-cGMP-PKG pathways, up-regulating MLC phosphorylation, causing actin polymerization (Dudek and Garcia, 2001; Duan et al., 2017). A study also found that Dex could reduce calcium influx by activating $\alpha_{2}$ receptors (Zhao et al., 2013). Therefore, we speculated that Dex might inhibit $\mathrm{Ca}^{2+}$ influx by activating $\alpha_{2}$ receptors and blocking $\mathrm{Ca}^{2+} / \mathrm{CaM}-\mathrm{PKC}$ and $\mathrm{Ca}^{2+}$-cGMP -PKG pathway, thereby inhibiting actin polymerization, but the specific mechanism needs to be further studied.

Besides ER-MITO contact, Drp1 activates and translocates from the cytoplasm to the mitochondrial pre-constriction site and cleaves the mitochondria. Present study found that Dex inhibits mitochondrial division through Drp1 phosphorylation. Dex could effectively inhibit the phosphorylation of Drp1 Ser616 in VECs and reduce the translocation of Drp1 from the cytoplasm to the mitochondria. As an important mitochondrial-related protein, Drp1 is of great significance to the regulation of mitochondrial structure and function. Also, Drp1 is known as DNM1L and DLP1, which was first reported in 1997 (Shinh et al., 1997), mainly distributed in the cytoplasm in the form of polymers under physiological condition. The activated Drp1 translocates from the cytoplasm to the mitochondrial outer membrane and polymerizes to form a ring-like structure that enables mitochondrial division. Besides, our prediction of iGPS and previous studies (Jiang H. K. et al., 2014; Prieto et al., 2016) showed that the activity of Drp1 Ser616 is mainly regulated by the ERK1/2 pathway. In the present study, Dex had a significant inhibitory effect on the phosphorylation of ERK1/2 in VECs after sepsis, which is consistent with previous studies (Jiang L. et al., 2014; Qiu et al., 2020). Meanwhile, we confirmed that both Dex and ERK1/2 inhibitor (PD98059) could effectively suppress the phosphorylation of Drp1 Ser616 in VECs, and inhibit vascular leakage, indicating that Dex may regulate the activation and translocation of Drp1 by inhibiting the ERK1/2 phosphorylation pathway. As multiple phosphorylation sites of Drp1 are activated to regulate its function after sepsis, we also observed that Dex could elevate the phosphorylation level of Ser637, which can inhibit the translocation of Drp1. Whether Dex can regulate Drp1 phosphorylation of other sites and the associated mechanism needs to be further studied. The present study demonstrated that Dex alleviated mitochondrial fission for sepsis by two key steps of mitochondrial fission. The relationship between two steps of Dex-protecting mitochondrial fission needs further investigation.

Although our experiments demonstrated that Dex protected the vascular endothelial barrier function by inhibiting mitochondrial division, there are some limitations that require further investigation. First, the present study verified the protective role of Dex in septic rats, and LPS stimulated VECs, but these models cannot fully simulate the complex pathophysiological environment in the human body. It is unknown whether Dex has a consistent protective function in large animals and humans. Second, We didn't investigate the mechanism of ERK1/2 inhibition regulated by Dex, which some studies suggest is independent of $\alpha_{2}$-adrenoceptor but related to TLR4/MyD88/MAPK/NF- $\mathrm{B}$ signaling pathway (Zhang et al., 2015). The detailed mechanisms need further exploration in the future. Moreover, we demonstrated the protective effect of Dex on VECs. However, in sepsis, the vascular smooth muscle cells (VSMCs) are less responsive to vasoactive drugs. Whether Dex can improve VSMCs responsiveness to vasoactive drugs and the mechanism must be further researched.

In conclusion, the present study demonstrated that Dex has a significant protective effect on the vascular endothelial barrier function of septic rats. The mechanism is related to inhibition of mitochondrial fission and protection of mitochondrial function through regulating the phosphorylation of Drp1 and reducing ER-MITO contacts of VECs via reducing actin polymerization (Figure 7). This research provides a potential therapeutic target for the treatment of sepsis.

\section{DATA AVAILABILITY STATEMENT}

The raw data supporting the conclusions of this article will be made available by the authors, without undue reservation.

\section{ETHICS STATEMENT}

The animal study was reviewed and approved by the Laboratory Animal Welfare and Ethics Committee of the Army Medical University.

\section{AUTHOR CONTRIBUTIONS}

TL, YH, LL, and HS conceived and designed the study and analyzed the data. HS and HD drafted the manuscript. TL and $Y Z$ revised the manuscript. TL acquired the financial support. All authors performed the experimental procedures.

\section{FUNDING}

This work was supported by the Key Program of the National Natural Science Foundation of China (Grant No. 81730059).

\section{SUPPLEMENTARY MATERIAL}

The Supplementary Material for this article can be found online at: https://www.frontiersin.org/articles/10.3389/fcell.2021. 636327/full\#supplementary-material

Supplementary Figure 1 | The effects of dexmedetomidine on the mitochondrial fusion of vascular endothelial cells after sepsis. (A-D) Western blot analysis of OPA1, Mfn1 and Mfn2 in VECs after sepsis, $n=3$. Normal, normal group; LPS, Ips group; Dex, dexmedetomidine group. 


\section{REFERENCES}

Allen, J. M., Field, C., Shoulders, B. R., and Voils, S. A. (2019). Recent updates in the pharmacological management of sepsis and septic shock: a systematic review focused on fluid resuscitation, vasopressors, and corticosteroids. Ann. Pharmacother. 53, 385-395. doi: 10.1177/1060028018812940

Armstrong, S. M., Mubareka, S., and Lee, W. L. (2013). The lung microvascular endothelium as a therapeutic target in severe influenza. Antiviral Res. 99, 113-118. doi: 10.1016/j.antiviral.2013.05.003

Brooks, C., Wei, Q., Cho, S. G., and Dong, Z. (2009). Regulation of mitochondrial dynamics in acute kidney injury in cell culture and rodent models. J. Clin. Invest. 119, 1275-1285. doi: 10.1172/JCI37829

Chakrabarti, R., Ji, W. K., Stan, R. V., de juan Sanz, J., Ryan, T. A., and Higgs, H. N. (2018). INF2-mediated actin polymerization at the ER stimulates mitochondrial calcium uptake, inner membrane constriction, and division. J. Cell Biol. 217, 251-268. doi: 10.1083/jcb.201709111

Duan, C., Kuang, L., Xiang, X., Zhang, J., Zhu, Y., Wu, Y., et al. (2020). Activated Drp1-mediated mitochondrial ROS influence the gut microbiome and intestinal barrier after hemorrhagic shock. Aging 12, 1397-1416. doi: 10. 18632/aging. 102690

Duan, C., Zhang, J., Wu, H. L., Li, T., and Liu, L. M. (2017). Regulatory mechanisms, prophylaxis and treatment of vascular leakage following severe trauma and shock. Mol. Med. Res. 4:11. doi: 10.1186/s40779-017-0117-6

Dudek, S. M., and Garcia, J. G. (2001). Cytoskeletal regulation of pulmonary vascular permeability. J. Appl. Physiol. 91, 1487-1500. doi: 10.1152/jappl.2001. 91.4.1487

Eismann, T., Huber, N., Shin, T., Kuboki, S., Galloway, E., Wyder, M., et al. (2009). Peroxiredoxin-6 protects against mitochondrial dysfunction and liver injury during ischemia-reperfusion in mice. Am. J. Physiol. Gastrointest. Liver Physiol. 296, 266-274. doi: 10.1152/ajpgi.90583.2008

Friedman, J. R., Lackner, L. L., West, M., Dibenedetto, J. R., Nunnari, J., and Voeltz, G. K. (2011). ER tubules mark sites of mitochondrial division. Science 334, 358-362. doi: 10.1126/science. 1207385

Gao, Z. J., Li, Y. Y., Wang, F., Huang, T., Fan, K. Q., Zhang, Y., et al. (2017). Mitochondrial dynamics controls anti-tumour innate immunity by regulating CHIP-IRF1 axis stability. Nat. Commun. 8:1805. doi: 10.1038/s41467-01701919-0

Hall, A. M., Rhodes, G. J., Sandoval, R. M., Corridon, P. R., and Molitoris, B. A. (2013). In vivo multiphoton imaging of mitochondrial structure and function during acute kidney injury. Kidney Int. 83, 72-83. doi: 10.1038/ki.20 12.328

Hatch, A. L., Gurel, P. S., and Higgs, H. N. (2014). Novel roles for actin in mitochondrial fission. J .Cell Sci. 127, 4549-4560. doi: 10.1242/jcs.153791

Jawad, I., Luksic, I., and Rafnsson, S. B. (2012). Assessing available information on the burden of sepsis: global estimates of incidence, prevalence and mortality. J. Glob. Health 2:010404. doi: 10.7189/jogh.02.010404

Jiang, H. K., Wang, Y. H., Sun, L., He, X., Zhao, M., Feng, Z. H., et al. (2014). Aerobic interval training attenuates mitochondrial dysfunction in rats postmyocardial infarction: roles of mitochondrial network dynamics. Int. J .Mol. Sci. 15, 5304-5322. doi: 10.3390/ijms15045304

Jiang, L., Li, L., Shen, J., Qi, Z., and Guo, L. (2014). Effect of dexmedetomidine on lung ischemia-reperfusion injury. Mol. Med. Rep. 9, 419-426. doi: 10.3892/ mmr.2013.1867

Koh, I. H., Menchaca-Diaz, J. L., Koh, T. H., Souza, R. L., Shu, C. M., Rogerio, V. E., et al. (2010). Microcirculatory evaluation in sepsis: a difficult task. Shock 34, 27-33. doi: 10.1097/SHK.0b013e3181e7e80c

Liu, Y., Kalogeris, T., Wang, M., Zuidema, M. Y., Wang, Q., Dai, H., et al. (2012). Hydrogen sulfide preconditioning or neutrophil depletion attenuates ischemiareperfusion-induced mitochondrial dysfunction in rat small intestine. Am. J. Physiol. Gastrointest. Liver Physiol. 302, 44-54. doi: 10.1152/ajpgi.00413.2010

Miranda, M. L., Balarini, M. M., and Bouskela, E. (2015). Dexmedetomidine attenuates the microcirculatory derangements evoked by experimental Sepsis. Anesthesiology 122, 619-630. doi: 10.1097/ALN.0000000000000491

Nelson, L. E., Lu, J., Guo, T. Z., Saper, C. B., Franks, N. P., and Maze, M. (2003). The alpha2-adrenoceptor agonist dexmedetomidine converges on an endogenous sleep-promoting pathway to exert its sedative effects. Anesthesiology 98, 428436. doi: 10.1097/00000542-200302000-00024
Page, A. V., and Liles, W. C. (2013). Biomarkers of endothelial activation/dysfunction in infectious diseases. Virulence 4, 507-516. doi: $10.4161 /$ viru. 24530

Peake, S. L., Delaney, A., Bailey, M., Bellomo, R., Cameron, P. A., Copper, D. J., et al. (2014). Goal-directed resuscitation for patients with early septic shock. N. Engl. J. Med. 371, 1496-1506. doi: 10.1056/NEJMoa1404380

Prieto, J., Leon, M., Ponsoda, X., Sendra, R., Bort, R., Ferrer-Lorente, R., et al. (2016). Early ERK1/2 activation promotes DRP 1-dependent mitochondrial fission necessary for cell reprogmmming. Nat. Commun. 7:11124. doi: 10.1038/ ncomms 11124

Qiu, Z., Lu, P., Wang, K., Zhao, X., Li, Q., Wen, J., et al. (2020). Dexmedetomidine inhibits neuroinflammation by altering microglial M1/M2 polarization through MAPK/ERK pathway. Neurochem. Res. 45, 345-353. doi: 10.1007/s11064-01902922-1

Rowland, A. A., and Vorletz, G. K. (2012). Endoplasmic reticulum-mitochondria contacts: function of the junction. Nat. Rev. 13, 601-615. doi: 10.1038/nrm3440

Roy, S., Kim, D., and Sankaramoorthy, A. (2019). Mitochondrial structural changes in the pathogenesis of diabetic retinopathy. J. Clin. Med. 8:1363. doi: 10.3390/ jcm8091363

Schmidt, K., Hernekamp, J. F., Philipsenburg, C., Zivkovic, A. R., Brenner, T., and Hofer, S. (2015). Time-dependent effect of clonidine on microvascular permeability during endotoxemia. Microvasc. Res. 101, 111-117. doi: 10.1016/j. mvr.2015.07.002

Sha, J., Zhang, H., Zhao, Y., Feng, X., Hu, X., Wang, C., et al. (2019). Dexmedetomidine attenuates lipopolysaccharide-induced liver oxidative stress and cell apoptosis in rats by increasing GSK-3 $3 / \mathrm{MKP}-1 / \mathrm{Nrf} 2$ pathway activity via the $\alpha_{2}$ adrenergic receptor. Toxicol. Appl. Pharmacol. 364, 144-152. doi: 10.1016/j.taap.2018.12.017

Shinh, H. W., Shinotsuka, C., Torii, S., Murakami, K., and Nakayama, K. (1997). Identification and subcellular localization of a novel mammalian dynaminrelated protein homologous to yeast Vps1p and Dnmlp. J. Biochem. 122, 525-530. doi: 10.1093/oxfordjournals.jbchem.a021784

Sun, Y. B., Zhao, H., Mu, D. L., Zhang, W., Cui, J., Wu, l., et al. (2019). Dexmedetomidine inhibits astrocyte pyroptosis and subsequently protects the brain in in vitro and in vivo models of sepsis. Cell Death Dis. 10:167. doi: 10.1038/s41419-019-1416-5

Valente, A. J., Maddalena, L. A., Robb, E. 1., Moradi, F., and Stuart, J. A. (2017). A simple ImageJ macro tool for analyzing mitochondrial network morphology in mammalian cell culture. Acta Histochem. 119, 315-326. doi: 10.1016/j.acthis. 2017.03.001

Vincent, J. L., Opal, S. M., Marshall, J. C., and Tracey, K. J. (2013). Sepsis definitions: time for change. Lancet 381, 774-775. doi: 10.1016/S0140-6736(12)61815-7

Wales, P., Schuberth, C. E., Aufschnaiter, R., Fels, J., Garcia-Aguilar, L., Janning, A., et al. (2016). Calcium-mediated actin reset (CaAR) mediates acute cell adaptations. Elife 5:19850. doi: 10.7554/eLife.19850

Wang, C. W., Shui, K., Ma, S. S., Lin, S. F., Zhang, Y., Wen, B., et al. (2020). Integrated omics in Drosophila uncover a circadian kinome. Nat.Commun. 11:2710. doi: 10.1038/s41467-020-16514-z

Wang, Y., Mattson, M. P., and Furukawa, K. (2002). Endoplasmic reticulum calcium release is modulated by actin polymerization. J. Neurochem. $82,945-$ 952. doi: 10.1046/j.1471-4159.2002.01059.x

Wu, W., Lin, C., Wu, K., Jiang, L., Wang, X., Li, W., et al. (2016). FUNDC1 regulates mitochondrial dynamics at the ER-mitochondrial contact site under hypoxic conditions. EMBO J. 35, 1368-1384. doi: 10.15252/embj.201593102

Yuan, F., Fu, H., Sun, K., Wu, S., and Dong, T. (2017). Effect of dexmedetomidine on cerebral ischemia-reperfusion rats by activating mitochondrial ATPsensitive potassium channel. Metab. Brain Dis. 32, 539-546. doi: 10.1007/ s11011-016-9945-4

Zhang, J., Wang, J., Luan, T., Zuo, Y., Chen, J., Zhang, H., et al. (2019). Deubiquitinase USP9X regulates the invasion of prostate cancer cells by regulating the ERK pathway and mitochondrial dynamics. Oncol. Rep. 41, 3292-3304. doi: 10.3892/or.2019.7131

Zhang, J., Wang, Z., Wang, Y., Zhou, G., and Li, H. (2015). The effect of dexmedetomidine on inflammatory response of septic rats. BMC Anesthesiol. 15:68. doi: 10.1186/s12871-015-0042-8

Zhao, H., Zhu, Y., Zhang, J., Wu, Y., Xiang, X., Zhang, Z., et al. (2020). The beneficial effect of HES on vascular permeability and its relationship with 
endothelial glycocalyx and intercellular junction after hemorrhagic shock. Front. Pharmacol. 11:597. doi: 10.3389/fphar.2020.00597

Zhao, J., Zhou, C. L., Xia, Z. Y., and Wang, L. (2013). Effects of dexmedetomidine on L-Type calcium current in rat ventricular myocytes. Acta Cardiol. Sin. 29, 175-180. doi: 10.1016/j.pcad.2013.01.003

Zhao, L., Zhuang, J., Wang, Y., Zhou, D., Zhao, D., Zhu, S., et al. (2019). Propofol ameliorates $\mathrm{H} 9 \mathrm{C} 2$ cells apoptosis induced by oxygen glucose deprivation and reperfusion injury via inhibiting high levels of mitochondrial fusion and fission. Front. Pharmacol. 10:61. doi: 10.3389/fphar.2019.00061

Zheng, D., Zhang, J., Zhang, Z., Kuang, L., Zhu, Y., Wu, Y., et al. (2020). Endothelial microvesicles induce pulmonary vascular leakage and lung injury during sepsis. Front. Cell Dev. Biol. 8:643. doi: 10.3389/fcell.2020. 00643

Zhu, Y., Wu, H., Wu, Y., Zhang, J., Peng, X., Zang, J., et al. (2016). Beneficial effect of intermedin 1-53 in septic shock rats: contributions of Rho kinase and Bkca pathway-mediated improvement in cardiac function. Shock 46, 557-565. doi: $10.1097 /$ SHK.0000000000000639
Zi, S. F., Li, J. H., Liu, L., Deng, C., Ao, X., Chen, D. D., et al. (2019). Dexmedetomidine-mediated protection against septic liver injury depends on TLR4/MyD88/NF-KB signaling downregulation partly via cholinergic antiinflammatory mechanisms. Int. Immunopharmacol. 76:105898. doi: 10.1016/j. intimp.2019.105898

Conflict of Interest: The authors declare that the research was conducted in the absence of any commercial or financial relationships that could be construed as a potential conflict of interest.

Copyright $\odot 2021$ She, Zhu, Deng, Kuang, Fang, Zhang, Duan, Ye, Zhang, Liu, Hu and Li. This is an open-access article distributed under the terms of the Creative Commons Attribution License (CC BY). The use, distribution or reproduction in other forums is permitted, provided the original author(s) and the copyright owner(s) are credited and that the original publication in this journal is cited, in accordance with accepted academic practice. No use, distribution or reproduction is permitted which does not comply with these terms. 